\title{
Sylvain Gatelais \\ Chapitre 14 Absence, manque et espace : quelques remarques sur la préposition without
}

\begin{abstract}
Absences are not events. They are not anything: where an absence is, there is nothing relevant there at all. Absences are bogus entities. Yet the proposition that an absence occurs is not bogus. David Lewis, « Causation as Influence », Journal of Philosophy 97.
\end{abstract}

\section{Introduction}

Si la plupart des contributions au présent volume ont jusqu'ici abordé la notion du manque à travers le prisme du signifié verbal, cette étude propose de s'intéresser à une autre partie du discours, souvent considérée comme moins lexicale ou signifiante, à savoir la préposition. C'est sans doute la définition (pour le moins succincte) que le Merriam-Webster donne de la préposition anglaise without qui a attiré notre attention et qui a été le point de départ de cette étude : used as a function word to indicate the absence or lack of something or someone. La tradition grammaticale, elle, utilisera assez souvent le terme d'abessif, à l'origine employé dans la description des langues finno-ougriennes (en particulier du finnois), pour désigner ce type de relation. Il s'agit dans ces langues d'un cas qui exprime « l'absence de quelque chose ${ }^{1} »$.

L'un des objectifs de cette étude sera donc de confronter la préposition avec d'autres marqueurs ou lexèmes exprimant le manque. S'il fallait rapprocher la préposition without de l'un des emplois du verbe manquer identifiés dans le présent volume (voir le questionnaire commun de base), il s'agirait ainsi sans équivoque du type 2 (« objet manquant, procès manquant : effet de sens d'absence ou de besoin »). Les énoncés du corpus entrant dans cette catégorie peuvent être traduits par le verbe to lack (ou plus rarement to miss) mais également par des structures attributives adjectivales be missing / short of... Deux prépositions pourraient

1 «A term used in grammatical description to refer to a type of inflection which expresses the meaning of absence, such as would be expressed in English by the preposition "without" " (Crystal, 2008).

https://doi.org/10.1515/9783110727609-014 
également figurer dans cette liste : without et out of. Aucune étude portant sur les prépositions ne peut complètement éluder la question du spatial dans la sémantique prépositionnelle, quelle que soit la réponse qu'on lui apporte. L'anglais nous y invite d'autant plus que c'est l'une des rares langues indo-européennes qui utilisent des prépositions dont le sens spatial est encore largement attesté. Il conviendra donc inévitablement de s'interroger sur les rapports qu'entretiennent l'abessif et la notion d'extériorité telle qu'elle peut être encore exprimée par out of et without. L'autre angle d'attaque possible est la négation : without a cette particularité d'être la seule préposition à laquelle on attribue une valeur négative. Celle-ci est également souvent assignée aux lexèmes exprimant le manque, comme l'a suggéré Alain Delplanque dans le présent volume.

Il convient avant tout de noter que without, en dépit de l'intérêt croissant porté ces dernières années aux marqueurs prépositionnels (en particulier sous l'impulsion de la grammaire cognitive), n'a fait l'objet d'aucune étude d'envergure. Il n'en va pas de même de sans, qui a intéressé un certain nombre de linguistes francisants, la plupart l'opposant à d'autres marqueurs (essentiellement avec ou bien les adverbes de manière). Citons entre autres les travaux d'I. Choi-Jonin et F. Mignon, de M. Riegel, de C. Molinier, de M-F. Lagacé ou encore de S. Feigenbaum cités en bibliographie. Si cette étude s'appuiera en grande partie sur leurs travaux, il faut noter d'emblée que certaines des propriétés distributionnelles de la préposition française ne sont pas transposables à l'anglais. On observe ainsi dans cette langue des contraintes sur la détermination du régime (l'article Ø étant souvent utilisé, là où l'anglais utilisera une détermination indéfinie : Il est sorti sans Ø parapluie / He went out without an umbrella), la présence d'un ne explétif dans les propositions introduites par sans que (mais condamnée par la grammaire normative et l'Académie française : Je suis sorti sans qu'il (ne) le sache), l'emploi du subjonctif dans des propositions finies, etc. À l'inverse, without présente des caractéristiques diachroniques, morphologiques et distributionnelles que l'on ne trouve pas chez son homologue français (ou dans la plupart des autres langues) : une morphologie à première vue motivée (bien que démentie par la diachronie, comme on le verra) par son antonymie avec with, un sens spatial encore attesté, une apparition tardive dans l'histoire de l'anglais, un système d'oppositions avec des lexèmes dérivés (adjectifs suffixés en -less ou composés avec -free), l'apparente équivalence entre with no $+\mathrm{N} /$ without + GN, l'absence de conjonction introduisant une proposition avec un verbe fini (without + V-ING / * without that $+\mathrm{P}$ ), etc. La plupart de ces différences, dont certaines nourriront notre réflexion, suggèrent que without mérite un traitement à part.

Afin de mener à bien cette étude, nous sommes parti du questionnaire commun de base (QCB) élaboré par les participants à l'opération de recherche «Analyse et formalisation de l'expression du manque» au sein de l'équipe 
«Sémantique Énonciative et Typologie des Langues » (LLL Tours). Le QCB nous a été particulièrement utile pour étayer notre réflexion sur les rapports qu'entretient without avec l'expression du manque. Nous avons ensuite utilisé différents corpus, en particulier le COCA (Corpus of Contemporary American English), créé par Mark Davies et constitué d'œuvres de fiction, d'articles de presse, de retranscriptions d'interviews télévisées et d'écrits académiques. Les données diachroniques ont été réunies grâce à l'Oxford English Dictionary (OED) et au Middle English Compendium en ligne.

\section{Situation effective et situation attendue}

Voici la définition que donne le Larousse du mot absence : « fait pour quelqu'un, quelque chose de ne pas se trouver à l'endroit où l'on s'attend à ce qu'il soit ». Si l'on examine maintenant la signification du verbe manquer, il s'agit, toujours selon le Larousse, de «ne pas disposer de choses ou d'êtres en quantité suffisante ou ne pas les avoir du tout ». Les deux notions semblent donc de prime abord assez voisines. Dans les deux cas, il y a le constat d'un décalage entre une situation idéale (et donc virtuelle, invalidée) souhaitée, attendue ou encore jugée nécessaire et l'état effectif des choses. En termes plus énonciatifs, comme le souligne Alain Delplanque dans le présent volume, nous avons une relation de repérage entre un terme A et un terme B. Les deux types de relations impliquent ainsi un « siège » (le terme $A$ ), la plupart du temps clairement exprimé dans l'énoncé, ainsi qu'un item manquant (le terme B). Ainsi, dans :

(1) Depuis cinquante-quatre ans que j'habite cette planète-ci, je n'ai été dérangé que trois fois. La première fois ç'a été, il y a vingt-deux ans, [...]. La seconde fois ç'a été, il y a onze ans, par une crise de rhumatisme. Je manque d'exercice. Je n'ai pas le temps de flâner. Je suis sérieux, moi (QCB).

... ce siège sera en l'occurrence le référent du sujet syntaxique ${ }^{2} j e$. L'item manquant sera quant à lui le référent du complément d'objet exercice. Il y a bien un décalage entre deux situations inférables à partir de cet énoncé : une situation effective (j'ai besoin d'une certaine quantité d'exercice, sans que celle-ci soit précisée) et une situation idéale (cette quantité devrait être supérieure, sans que

2 Mais comme le souligne Alain Delplanque, ce n'est pas toujours le cas avec ce verbe en français. 
celle-ci soit précisée). On retrouve cette configuration dans un énoncé contenant la préposition abessive without :

(2) He had gone out without his parents' permission. «Il était sorti sans la permission de ses parents ».

(3) It was not a nice house; it was a house without a garden in the middle of a noisy street. (Virginia Woolf, A Room of One's Own and Three Guineas).

"Ce n'était pas une jolie maison : c'était une maison sans jardin au milieu d'une rue bruyante».

Dans ces deux énoncés, l'item manquant est le référent du régime de la préposition without (a garden, his parents' permission). Le siège peut être de différentes natures. Dans l'énoncé (3), nous avons un syntagme nominal dont la tête est house postmodifiée par le syntagme prépositionnel. Le siège du manque sera donc le référent de cette tête. Dans l'énoncé (2), le siège du manque est le procès correspondant à $<$ he / go out $>$. Without est donc le terme relateur qui marquera explicitement dans l'énoncé cette absence. Il y a bien, par ailleurs, un décalage constaté entre une situation effective et une situation attendue / virtuelle, invalidée en ceci que les énoncés en without sont présupposants.

\subsection{Without et la présupposition}

Cette propriété est suggérée par la définition donnée plus haut par le Larousse ( "où l'on s'attend à ce qu'il soit ») et a été observée par certains linguistes pour la préposition sans. Ainsi, pour Riegel, « lorsqu'un locuteur emploie la préposition sans ou la conjonction sans que, il prend pour acquis que l'implication niée par la locution est concevable par son interlocuteur» (Riegel, 1977, p. 348). Pour Lagacé, « lorsque nous énonçons A sans B, c'est que nous nous attendons habituellement à B » (Lagacé, 1987, p. 172). Ce qui est vrai pour sans l'est globalement pour without en anglais : cette propriété semble opérante avec tous les effets de sens que nous verrons plus bas (instrumental, comitatif, relation postnominale du type $\mathrm{X}$ doesn't have $\mathrm{Y}$...). Cette présupposition d'existence peut être induite soit par la connaissance du monde de l'énonciateur :

(4) Last year a baby was born without eyes in Denver, Colorado.

«L’an dernier, un bébé est né sans yeux à Denver, Colorado » (un bébé a normalement des yeux). 
(5) These cookies are made without flour.

«Ces cookies sont préparés sans farine » (on prépare normalement des cookies avec de la farine).

soit par le cotexte :

(6) We passed two ruined abbeys, one with a tower and one without.

« Nous sommes passés devant deux abbayes en ruine, l'une avec une tour et l'autre sans. »

(7) Only two kinds of people exist - those with children and those without (COCA).

«Il existe seulement deux catégories de personnes : celles avec des enfants et celles qui n'en ont pas » (mot à mot : et celles sans).

Ainsi, hors contexte, un énoncé contenant un without à valeur instrumentale tel que :

(8) ?Mark opened the door without an ice pick.

« Mark a ouvert la porte sans pic à glace. »

est difficilement recevable, car, prototypiquement, un pic à glace n'a pas pour fonction d'ouvrir une porte et donc de servir d'instrument à la réalisation du procès open a door. Les énoncés suivants seront en revanche tout à fait acceptables :

(9) He was able to climb the mountain without an ice pick.

« Il a pu escalader la montagne sans pic à glace » (un pic à glace peut servir à escalader une montagne).

(10) I got locked in my basement. The only tools I had were a hammer and an ice pick $_{i}$. Luckily, I managed to open the door without the ice pick .

« J'ai été enfermé dans mon sous-sol. Les seuls outils que j'avais à ma disposition étaient un marteau et un pic à glace. Heureusement, j'ai réussi à ouvrir la porte sans le pic à glace. »

Dans l'énoncé (10), comme en atteste l'emploi du déterminant défini THE, qui a ici un rôle anaphorique, le cotexte permet d'envisager et de préconstruire la relation prédicative $<\mathrm{I} /$ open the door with an ice pick $>$ qui semble dès lors, sinon attendue, du moins envisageable. 
Notons au passage qu'à l'inverse, l'emploi de la préposition with sera difficilement recevable (ou créera un effet pléonastique) si la relation entre repère et repéré est nécessaire ou " évidente », en particulier lorsque with exprime une relation partie-tout (surtout en contexte postnominal) ou instrumentale :

(11) ??He kissed his wife with his lips.

«Il a embrassé sa femme avec ses lèvres. »

(12) ??He slapped his son with his hand.

«Il a giflé son fils avec sa main. »

(13) ??He paid with money.

« Il a payé avec de l'argent. »

(14) ??a man with a head / eyes / feet etc.

« un homme avec une tête / des yeux / des pieds, etc. »

L'emploi de without comme relateur dans la relation $<\mathrm{A} r \mathrm{~B}>$ implique donc qu'il y ait une compatibilité ou une congruence entre le repère et le repéré, cette congruence pouvant être soit notionnelle, soit générée par un contexte spécifique (linguistique et / ou extralinguistique). Cette propriété suggère donc que, pour bien appréhender le rôle et les effets de sens exprimés par without, il faudra jauger l'adéquation entre $A$ et $B$ en prenant en considération les types de référents désignés par ces deux entités : réalisation d'un procès dont il faudra préciser le type (action, état, télicité), type de noms (dense, discret, compact), type de référents (humain, non humain), etc. Ceci suppose également qu'il faille examiner d'autres marqueurs présents dans l'énoncé (le cotexte étroit), tels que le type de détermination du nom régime ou l'aspect verbal par exemple, mais également le cotexte plus large et la situation.

\subsection{Pourquoi without ne peut-elle introduire une proposition finie ?}

La nature présupposante de without pourrait nous aider à comprendre une particularité morpho-syntaxique de l'anglais. Contrairement à ce que l'on peut observer dans un certain nombre de langues, il n'est pas possible en anglais de construire une proposition subordonnée avec un verbe fini introduite par without. Là où le français, l'espagnol ou l'italien utiliseront une locution conjonctive telle que sans que, sin que, ou senza che suivie du subjonctif, l'anglais aura obligatoirement 
recours à une nominalisation gérondive en V-ING introduite par without, avec un sujet exprimé ou non :

(15) No hago nada sin que lo sepas.

(16) Je ne fais rien sans que tu (ne) le saches.

(17) Non faccio niente senza che tu lo sappia.

(18) I do nothing without your / you knowing it ( ${ }^{*}$ without that you know it).

Dans toutes ces constructions, il s'agit de nier un procès concomitant (voir § 2) à celui de la principale. On sera attentif à l'emploi du subjonctif qui a ici sa valeur attendue : le procès décrit dans la subordonnée n'est pas actualisé et est simplement envisagé, du fait de l'orientation négative de la conjonction.

On constate donc que l'anglais a opté dans son histoire pour une tout autre stratégie structurale et morphosyntaxique. Toutefois, cela n'a pas toujours été le cas, comme en attestent les exemples suivants :

(19) The speres flew in peces wythout that ony of theym felle to the grounde. (The right plesaunt and goodly historie of the foure sonnes of Aymon, 1489)

« Les lances se brisèrent en morceaux en plein vol sans qu'aucun d'eux ne touchât le sol.»

(20) This seem'd to be done, without that the King was fully inform'd thereof. (Edward Herbert Baron Herbert of Cherbury, The Life and Reigne of King Henry the Eighth, 1649)

«Il semblerait que cela ait été accompli, sans que le roi n’en fût pleinement informé.»

La locution conjonctive semble être attestée dès le $\mathrm{xv}^{\mathrm{e}}$ siècle et avait en réalité deux sens possibles en fonction du mode verbal utilisé dans la subordonnée. Lorsque le verbe était utilisé à l'indicatif, comme dans les exemples ci-dessus, la conjonction signifiait « sans que » et indiquait une circonstance concomitante négative. Lorsque le verbe était employé au subjonctif (caractérisé par l'absence de -s de la $3^{\mathrm{e}}$ personne ou par la forme be à toutes les personnes), la proposition exprimait alors une condition négative et without signifiait alors « unless » (à moins que / if... not...). Le subjonctif s'imposait donc dans ce contexte, au même titre qu'il s'imposait à l'époque dans les propositions en if, dans la mesure où le procès était envisagé sur le plan fictif et avait une valeur contrefactuelle : 
(21) He schalle never have me to his make with owte he be baptizede and receyve the feithe of Criste. (Higden's Polychronicon, $\mathrm{xv}^{\mathrm{e}}$ siècle)

« Il ne m'aura jamais pour épouse à moins qu'il ne soit baptisé et reçoive la foi du Seigneur. »

(22) He may barke, but he cannot bite without a man come within his reach. (J. Shute, Judgement and Mercy, 1643)

« Peut-être qu'il aboie, mais il ne peut pas mordre à moins qu'un homme ne s'approche trop près de lui. »

Ces deux usages vont disparaître assez rapidement. Le premier, qui sera remplacé par la forme non finie en V-ING, semble être sorti de l'usage après le xviI ${ }^{\mathrm{e}}$ siècle. Le second, qui sera remplacé progressivement par unless, se maintiendra plus longtemps, au xviII ${ }^{\mathrm{e}}$ et au xix ${ }^{\mathrm{e}}$ siècle dans la langue parlée et familière, et même au-delà dans certaines formes dialectales de l'anglais (notamment américaines). Dans ce dernier cas, le subjonctif, qui était autrefois de règle, laissera place la plupart du temps à l'indicatif :

(23) Without a great change takes place the meeting is sure to commence to-morrow. (Daily News, 1887)

«À moins d'un grand changement, il est certain que la réunion commencera demain. »

(24) ... the artist, of whatever kind, cannot produce a truthful work without he understands the laws of the phenomena he represents... (Herbert Spencer, Intellectual, Moral, and Physical, 1891)

«... l'artiste, quel que soit son domaine, ne peut produire une œuvre sincère à moins de comprendre les lois qui régissent les phénomènes qu'il dépeint... »

(25) You don't know about me without you have read a book by the name of The Adventures of Tom Sawyer. (Mark Twain, The Adventures of Huckleberry Finn, 1884)

"Vous ne savez rien de moi à moins d'avoir lu un livre intitulé Les Aventures de Tom Sawyer. »

Deux raisons peuvent être avancées pour rendre compte de cette évolution. La première est la disparition progressive du subjonctif présent, amorcée à la fin de la période moyen-anglaise, due à ses trop grandes ressemblances morphologiques avec l'indicatif. S'il est encore courant au xvi ${ }^{\mathrm{e}}$ siècle, son usage ne cessera 
de reculer à partir du xviI e siècle. Comme le rappelle Vallins (1956, p. 29-30), l'emploi de ce mode était devenu tellement restreint aux xvII ${ }^{\mathrm{e}}$ et $\mathrm{xvIII}^{\mathrm{e}}$ siècles que cette forme verbale était devenue un véritable mystère pour les grammairiens de l'époque, donnant lieu au passage à des analyses pour le moins farfelues. Au xvII ${ }^{\mathrm{e}}$ siècle, le dramaturge et grammairien Ben Jonson (cité par Visser, 1966, p. 788) y voyait par exemple un pluriel, pensant que les noms au singulier s'accordaient avec un verbe au pluriel quand ils étaient précédés de certaines conjonctions (« some nouns, though singular [...] require a verb plurall-especially when the verbe is joined to an adverbe, or conjunction »). Joseph Priestley (1761, p. 15), qui reconnaissait que cette forme n'était guère plus usitée à son époque mais qu'elle était courante quelques générations auparavant ( our forefathers paid a very strict and scrupulous regard to it »), estimait qu'il s'agissait de l'ellipse d'un auxiliaire et que le verbe était à l'infinitif.

Afin de marquer la différence entre ces deux emplois de without (sans que et à moins que), l'opposition désormais caduque entre indicatif et subjonctif fut remplacée et compensée par l'opposition morphosyntaxique entre proposition à verbe fini et proposition à verbe non fini. Si l'infinitif est courant en espagnol, en français ou en allemand après sin, ohne ou sans :

(26) Il est parti sans dire un mot.

(27) Er ist gegangen, ohne ein Wort zu sagen.

... la syntaxe de l'anglais, elle, interdit l'emploi d'un infinitif après une préposition :

(28) Avant de partir, n'oublie pas de poster la lettre.

(29) ${ }^{*}$ Before to leave, remember to post the letter.

Ce n'est donc pas cette forme non finie du verbe qui a pu être retenue. Or, c'est précisément à partir $\mathrm{du} \mathrm{xvII}^{\mathrm{e}}$ siècle que l'anglais développera pleinement une «troisième voie » qui rendra possible le recours à une seconde forme non finie. Comme le souligne D. Boulonnais, c'est à cette époque que les nominalisations gérondives apparaissent « du fait des nouvelles possibilités de construction verbale qui leur permettaient d'entrer en concurrence directe avec l'infinitif ». Ces structures, émergentes aux xvI ${ }^{\mathrm{e}}$ et $\mathrm{xvII}^{\mathrm{e}}$ siècles, deviendront courantes aux xVII ${ }^{\mathrm{e}}$ et $x_{x x}{ }^{e}$ siècles, où elles adopteront un statut pleinement phrastique (Boulonnais, 2004, p. 80). 
Si certaines langues semblent donc privilégier, avec l'emploi du subjonctif et de l'infinitif, la non-actualisation du procès dans ce type de proposition, l'anglais, en optant exclusivement pour la forme en V-ING, semble avoir adopté une tout autre stratégie. Plusieurs linguistes énonciativistes ont ainsi vu dans cet opérateur la trace d'une préconstruction, d'un déjà ou d'une antériorité opérationnelle I psychogrammaticale (Lapaire et Rotgé, 1992). Adamczewski voit en V-ING un marqueur de phase 2 ou de thématicité (Adamczewski et Delmas, 1982). Denis Jamet évoque quant à lui « une stabilité notionnelle », précisant qu'« il y a insistance sur le sémantisme de la notion, contrairement à une forme plus verbale » (Jamet, 2008, p. 40). C'est donc sans doute la nature présupposante du without abessif (sans que) qui justifierait également le recours exclusif à la forme nominalisée du verbe, plus notionnelle que prédicationnelle et cantonnant cet opérateur dans la catégorie des prépositions. À l'inverse, le without de condition négative (unless), non présupposant, imposera le mode à verbe fini, plus orienté vers la prédication.

\section{Without, forme négative ou antonymique de with?}

Comme l'a observé J. Cervoni, on considère souvent que les prépositions forment des micro-systèmes binaires (Cervoni, 1991, p. 154). Ce qui semble vrai pour des couples tels dans / en et $\grave{a}$ / de l'est sans doute bien davantage pour le couple avec / sans ou with / without : la plupart des linguistes, des grammaires et des dictionnaires (y compris ceux qui n'adoptent pas une approche systémique du phénomène prépositionnel) les traitent en parallèle. Deux termes de métalangage relativement voisins reviennent ainsi régulièrement pour qualifier la relation sémantique qui unit les deux prépositions : antonymie et négation. Ainsi, pour Riegel et al., la négation du rapport exprimé par la préposition avec (à savoir la concomitance et la coprésence) est dénotée par «son antonyme sans » (Riegel et al., 1994, p. 372). Dans le cas de l'anglais, Quirk et al. voient en without « the negative of with ». Notons d'emblée que, comme le rappellent la plupart des manuels de lexicologie, le terme antonymie est un mot fourre-tout, susceptible de décrire plusieurs types de relations sémantiques. Ainsi, selon Crystal :

In its most general sense, [the term antonymy] refers collectively to all types of semantic oppositeness (antonyms), with various subdivisions then being made (e. g. between graded antonyms, such as big $\sim$ small, where there are degrees of difference, and ungraded antonyms, such as single $\sim$ married, where there is an either / or contrast) (Crystal, 2008, p. 28).

Il serait donc plus juste de parler dans le cas du couple with / without de complémentaires (« on dit que des unités lexicales sont complémentaires quand la 
négation de l'une dans un énoncé implique l'affirmation de l'autre, et inversement. » [Dubois et al., 2002, p. 106]). Without X serait donc a priori, d'un point de vue logique, l'équivalent de with $\neg \mathrm{X}$ (ou with no $\mathrm{X}$ ). De même, not without $\mathrm{X}$, devrait être équivalent à with $\mathrm{X}$ (selon le principe que $: \neg \neg p \Leftrightarrow p$ ). Cependant, d'un point de vue linguistique (aussi bien sémantique qu'énonciatif ou pragmatique), ces deux relations d'équivalence logique sont sans doute discutables.

Corblin et Tovena (2003) fournissent dans les langues romanes un certain nombre de critères permettant d'identifier la préposition sans comme relevant de l'expression de la négation (la présence d'une complémentation verbale en de + $\mathrm{N}$, coordination avec ni..., suite discursive spécifique en moi / toi, etc. non plus, etc.). Si ces critères sont pour la plupart inapplicables à l'anglais, il demeure possible et même assez aisé de mettre au jour un ensemble de tests analogues pour cette langue :

Tableau 1 : Propriétés négatives de without

\begin{tabular}{|c|c|}
\hline Critères & Exemple \\
\hline $\begin{array}{l}\text { A. La substitution de with no }+\mathrm{N} \text { à without est } \\
\text { la plupart du temps possible. }\end{array}$ & $\begin{array}{l}\text { (30) She ordered a coke without ice / with no } \\
\text { ice. "Elle a commandé un coca sans glace. " }\end{array}$ \\
\hline $\begin{array}{l}\text { B. Présence dans le régime de negatively-ori- } \\
\text { ented polarity-sensitive items (NPIs) }{ }^{3} \text { tels que } \\
\text { l'opérateur de parcours any (et tous ses déri- } \\
\text { vés : anyone, anywhere, any longer / more...), } \\
\text { much et many... Il peut s'agir aussi d'adverbes } \\
\text { ou d'adjoints tels que yet, ever, at all... ou } \\
\text { bien, dans les propositions gérondives intro- } \\
\text { duites par without, d'idiomes verbaux norma- } \\
\text { lement utilisés à la forme négative (hear / say } \\
\text { a word / a sound, lift a finger (to help), move a } \\
\text { muscle, see a (living) soul...). }\end{array}$ & $\begin{array}{l}\text { (31) She's surely managed to get on the train } \\
\text { this time, but without any money, or papers, or } \\
\text { luggage... (COCA) } \\
\text { "Elle est sans doute parvenue à monter dans } \\
\text { le train cette fois-ci, mais sans argent, ni pa- } \\
\text { piers, ni bagages. " } \\
\text { (32) The U. S. decided to send 100,000 troops } \\
\text { to Saudi Arabia without consulting anyone. } \\
\text { "Les États Unis ont décidé d'envoyer } 100000 \\
\text { hommes en Arabie Saoudite sans consulter } \\
\text { quiconque. " } \\
\text { (33) I could have easily ruined your reputation } \\
\text { and your teaching career without much effort. } \\
\text { (COCA) } \\
\text { "J'aurais pu facilement ruiner votre réputation } \\
\text { et votre carrière d'enseignant sans beaucoup } \\
\text { d'efforts. » } \\
\text { (34) You can have it all without lifting a finger. } \\
\text { (COCA) } \\
\text { "Tu peux tout avoir sans lever le petit doigt. » }\end{array}$ \\
\hline
\end{tabular}

3 Terminologie de Huddleston et Pullum (2002, p. 823). 


\begin{tabular}{|c|c|}
\hline Critères & Exemple \\
\hline $\begin{array}{l}\text { C. Without n'admet pas de négateur absolu } \\
\text { (absolute negators) dans son régime (en an- } \\
\text { glais standard du moins) tels que not, no (ou } \\
\text { ses dérivés : nowhere, nobody...), never, nei- } \\
\text { ther... Impossibilité également d'y trouver des } \\
\text { positively-oriented polarity-sensitive items } \\
\text { (PPIs) }{ }^{3} \text { tels que les quantifieurs few ou little, ou } \\
\text { certains adverbes (as well, too, already...) que } \\
\text { l'on trouve normalement exclusivement dans } \\
\text { la phrase affirmative }{ }^{4} \text {. }\end{array}$ & $\begin{array}{l}\text { (35) *She bought it without little money. } \\
(36) * \text { She bought it without no money. } \\
(37) * \text { He came without few people. } \\
(38) * \text { He left without my not knowing it. }\end{array}$ \\
\hline $\begin{array}{l}\text { D. Quand l'opérateur de négation NOT précède } \\
\text { without, le sens abessif disparaît et la prépo- } \\
\text { sition devient référentiellement synonyme de } \\
\text { with avec un effet souvent intensif (voir infra). }\end{array}$ & $\begin{array}{l}\text { (39) "I pawned it for } 20 \text { bucks" he says, not wi- } \\
\text { thout pride. (COCA) } \\
\text { " "Je l'ai mis en gage pour } 20 \text { billets", dit-il non } \\
\text { sans fierté. " } \\
\text { (40) The deal, however, is not without risks. } \\
\text { (COCA) } \\
\text { "La transaction, cependant, n'est pas sans } \\
\text { risque. » } \\
\text { (41) The man was not without charm. (COCA) } \\
\text { "Cet homme n'était pas dénué de charme. " }\end{array}$ \\
\hline
\end{tabular}

Pour autant, la négation ne porte pas sur l'ensemble de la prédication, comme le montrent les tests donnés par Klima (1964). Il s’agit donc bien d'une négation de constituant.

(42) 'He came to the party without his wife. / He opened the door without a knife.' 'So did I.' / *'Neither did I.'

\subsection{Manque, absence et négation}

Revenons à l'opération du manque qui nous intéresse dans le présent volume. Qu'est-ce qui peut rapprocher without d'un verbe exprimant le manque, tel que lack par exemple? Alain Delplanque rappelle que l'affirmation formelle de manquer renvoie à une négation notionnelle, et qu'il faut ranger ce verbe « avec les

4 Il est en revanche tout à fait possible de trouver l'un de ces opérateurs dans la prédication principale. Comparons : I never leave home without my purse. vs. Michelle Obama jabs at Trump without ever/*never mentioning his name. 
verbes de sens privatif comme : rater, louper, faillir, échouer, enfreindre, éviter de, oublier de, négliger de, etc. ». Toutefois, le statut négatif de lack semble moins clair. S'il partage en effet un certain nombre des caractéristiques vues dans le tableau 1, en particulier l'emploi de any, much et many dans son objet :

(43) The cramped house lacked any kind of intimacy. (COCA)

"Cette maison exiguë était dépourvue de tout type d'intimité. »

(44) Her husband, a former fisherman, worked in a fabric factory because he lacked any skills. (COCA)

"Son mari, un ancien pêcheur, travaillait dans une usine, faute de compétences » (mot à mot : car il manquait de compétences).

(45) The interior lacks much usable storage. (COCA)

«L'intérieur manque de beaucoup d'espace de rangement utilisable. »

(46) We lack many things. (COCA)

« Nous manquons de beaucoup de choses.»

(47) She doesn't lack self-confidence.

«Elle ne manque pas de confiance en elle.»

$=$ She has a lot of $/$ enough self-confidence.

«Elle a beaucoup / assez de confiance en elle. »

She lacks self-confidence.

«Elle manque de confiance en elle.»

= She has no / not enough confidence.

«Elle n'a pas / pas assez de confiance en elle.»

... certaines propriétés de Klima, telle que la polarité des tags ou des reprises elliptiques, semblent en revanche inopérantes :

(28) a- 'She lacks self-confidence.' 'So do I. / *Neither do I.'

b- She lacks self-confidence, doesn't she? / *does she?

On en conclura qu'on a affaire à une négation notionnelle, non marquée explicitement : la polarité de la phrase reste donc positive.

Une autre question que soulève l'étude en parallèle du marqueur abessif without et du verbe lack est la distinction entre la notion d'absence et celle de manque. 
(49) a- He lacks common sense.

« Il manque de bon sens. »

b- He's without common sense.

«Il est dépourvu de bon sens. »

L'énoncé (49-a) avec lack ne dit pas qu'il n'a pas du tout de bon sens. À l'inverse, l'énoncé (49-b) en without sera glosable par : He doesn't have any common sense. Par conséquent, without semble a priori constituer une véritable polarité en opposant le tout au rien, l'absence à la présence (qui serait, elle, exprimée au moyen de with) sans aucun état intermédiaire possible. Lack pourrait à l'inverse être glosé par not have enough ou run short of. Ceci ne semble pourtant pas systématique :

(50) They lived in appalling conditions, lacking even the most primitive sanitation. (COCA)

«Ils vivaient dans des conditions effroyables et manquaient même des installations sanitaires les plus rudimentaires. »

(51) Ron is without money.

« Ron est sans argent. »

L'énoncé (51) n'implique pas nécessairement que Ron n'ait pas du tout d'argent : il peut simplement vouloir dire qu'il n'en a pas assez ou en quantité jugée satisfaisante. Au contraire, en (50), l'idée de « quantité insuffisante » est absente ${ }^{5}$. Cette binarité entre le « tout » ou le « rien » que l'on attribue à without est également démentie par le fait que les groupes prépositionnels introduits par cette préposition peuvent être modifiés par des adverbes de degré du type entirely / completely, en particulier lorsque le nom régime a un fonctionnement indénombrable (mass noun) :

5 On sera d'ailleurs attentif au fait qu'aucun des énoncés tirés du Petit Prince dans le QCB n'est traduit par Howard Richard en anglais au moyen du verbe to lack. Le traducteur aura souvent recours à have no $+\mathrm{N}$ qui renvoie normalement à une quantité nulle : And the people have no imagination («Les gens manquent d'imagination»), They have no roots, and that makes their life very difficult («Ils manquent de racines, ça les gêne beaucoup »). 
(52) If the increased rate of melting continues, the summertime Arctic could be totally without ice by 2030, a date forty years earlier than previously anticipated.

«Si l'accélération du rythme de la fonte des glaces continue, l'Arctique pourrait être totalement dépourvu de glace en été d'ici 2030, 40 ans plus tôt que ce que les prévisions annonçaient jusqu'ici. »

(53) Can Job claim that he is utterly without sin?

« Job peut-il prétendre qu'il est totalement exempt de tout péché ?»

(54) His wife is totally without irony. (COCA)

«Sa femme est totalement dépourvue d'ironie.»

(55) Enid, with its population of 47,000, was entirely without electricity for days. «Enid, avec sa population de 47000 habitants, resta sans électricité du tout pendant des jours.»

Without peut donc indiquer un état intermédiaire entre l'absence totale et la présence. L'interprétation absence totale vs. absence partielle / quantité insuffisante sera ainsi générée par des paramètres contextuels. À ce titre, le type de nom (dense, compact, discret) et sa détermination auront un rôle à jouer : le verbe lack marquera plutôt l'absence totale lorsque son complément d'objet sera un nom discontinu au singulier par exemple.

Qu'est-ce qui différencie absence et manque? La nuance réside essentiellement dans le jugement dépréciatif de l'énonciateur : avec des verbes tels que miss, lack ou manquer, l'énonciateur stigmatise l'impact de l'absence de B sur le repère A, qui apparaît comme un détrimentaire (voir Delplanque dans le présent volume). Dire que La soupe manque de sel déprécie la soupe, de même que David's real problem is that he lacks confidence déprécie David (comme le suggère problem). De tels énoncés relèvent donc de la modalité de type 3 selon Culioli (la modalité évaluative axiologique) : l'énonciateur émet un jugement et sous-entend que l'absence du repéré $B$ a un impact négatif sur l'état du siège du manque A. Cette orientation modale semble faire défaut aux énoncés en without :

(56) I can see the sign without my glasses.

« Je peux voir le panneau sans mes lunettes.»

(57) He did his exercises without a calculator.

« Il a fait ses exercices sans calculatrice.» 
Ces énoncés ne sont que des constats. En (56), ni la réalisation du procès $<\mathrm{I} /$ see the sign>, ni l'état résultant du référent du sujet $I$ ne semblent ici dépréciés.

\subsection{L’asymétrie du système}

Si l'intuition et la ressemblance morphologique (sur laquelle nous reviendrons plus loin) nous encouragent à opposer with et without, force est de constater que le traitement lexicographique de ces deux prépositions ne reflète que très partiellement ce parallélisme. La plupart des dictionnaires se sont ainsi efforcés d'offrir des descriptions, certes souvent intuitives, mais relativement exhaustives des différents effets de sens de with. On dénombre ainsi 11 acceptions différentes dans l'entrée with du Merriam-Webster, elles-mêmes divisées en plusieurs sous-acceptions. L'entrée without dans ce même dictionnaire ne répertorie que deux effets de sens de la préposition : 1 . outside; 2 . used as a function word to indicate the $a b$ sence or lack of something or someone. On retrouve une dissymétrie analogue dans le Cambridge Dictionary, le Longman et la plupart des dictionnaires (exception faite de l'OED). Tandis que la lexicographie assigne traditionnellement à with des emplois multiples (variables d'un dictionnaire à l'autre : comitatif, instrumental, manière, simultanéité, coprésence...), la polysémie de without semble souvent traitée de manière moins éclatée, les dictionnaires privilégiant l'idée de « manque », d'« absence » ou de « négation » au détriment des relations circonstancielles du type accompagnement, instrument, manière etc. Les grammaires ne sont en général guère plus précises, même si $A$ Comprehensive Grammar of the English Language de Quirk et al. (1985) mentionne without comme négation de with dans les sections consacrées aux prépositions qui expriment la manière et l'instrumental.

Le tableau 2 tente donc de pallier l'absence d'étude comparative systématique entre les deux prépositions. Si with est le complémentaire de without, il devrait être possible de les substituer l'un à l'autre dans tous les contextes. Cette étude prend comme point de départ la distribution de la préposition with ainsi que ses effets de sens traditionnellement identifiés ${ }^{6}$ (Gatelais, 2008). Loin de viser

6 L'identification de ces effets de sens pour with / avec n'est pas sans poser de nombreux problèmes. Certains linguistes ont d'ailleurs proposé d'expliquer cette polysémie. Certains ont par exemple tenté d'identifier des emplois prototypiques dont dériveraient tous les autres : Schlesinger (1979) a expliqué l'emploi de manière en argumentant qu'il dériverait de l'emploi instrumental. Pour Lakoff et Johnson (1980), c'est l'idée d'accompagnement qui permettrait d'expliquer métaphoriquement l'emploi instrumental. D'autres linguistes ont, à l'inverse, essayé d'identifier une valeur invariante abstraite, sorte de dénominateur commun entre tous les emplois. Ainsi, pour 
une quelconque exhaustivité des contextes ou des effets de sens possibles, il s'agit ici de faire état de la symétrie (ou de l'asymétrie) éventuelle du système et d'identifier les zones où la complémentarité supposée des deux prépositions est mise à l'épreuve. Ce qui ressort de ces données, c'est globalement l'impossibilité de faire la substitution dans trois types de contextes :

Tableau 2 : Valeurs et portées de with et son remplacement par without

\begin{tabular}{|c|c|c|c|c|}
\hline $\begin{array}{l}\text { Portée } \\
\text { syntaxique }\end{array}$ & $\begin{array}{l}\text { Valeur } \\
\text { sémantique }\end{array}$ & $\begin{array}{l}\text { Contraintes } \\
\text { contextuelles }\end{array}$ & WITH & WITHOUT \\
\hline \multirow[t]{3}{*}{ Rection } & & $\begin{array}{l}\text { Complémentation } \\
\text { adjectivale } \\
\text { ADJ + with GN }\end{array}$ & $\begin{array}{l}\text { He's satisfied with } \\
\text { the job. } \\
\text { "Il est satisfait } \\
\text { de son travail. » }\end{array}$ & Non \\
\hline & $\begin{array}{l}\text { Le plus souvent } \\
\text { relation métony- } \\
\text { mique de partie / } \\
\text { tout, de posses- } \\
\text { sion etc. }\end{array}$ & $\begin{array}{l}\text { Complémentation } \\
\text { nominale } \\
\mathrm{N} \text { with / without } \\
\text { GN }\end{array}$ & $\begin{array}{l}\text { He talked to a } \\
\text { man with a scarf. } \\
\text { "Il a parlé à un } \\
\text { homme avec une } \\
\text { écharpe." }\end{array}$ & $\begin{array}{l}\text { a man without a } \\
\text { scarf } \\
\text { un homme sans } \\
\text { écharpe }\end{array}$ \\
\hline & & $\begin{array}{l}\text { Complément ver- } \\
\text { bal (en position } \\
\text { de second ou } \\
\text { troisième argu- } \\
\text { ment) } \\
\text { V + with GN (tran- } \\
\text { sitif indirect) } \\
\text { V + GN + with GN } \\
\text { (ditransitif) }\end{array}$ & $\begin{array}{l}\text { a) Stop fighting } \\
\text { with your brother! } \\
\text { "Arrête de te bat- } \\
\text { tre avec ton } \\
\text { frère. " } \\
\text { b) She feared she } \\
\text { wouldn't be able } \\
\text { to cope with two } \\
\text { new babies. } \\
\text { " Elle craignait de } \\
\text { ne pas être ca- } \\
\text { pable de s'en sor- } \\
\text { tir avec deux nou- } \\
\text { veaux nés. " } \\
\text { c) Mix the cream } \\
\text { with ketchup. } \\
\text { " Mélangez la } \\
\text { crème avec le ket- } \\
\text { chup. " }\end{array}$ & Non \\
\hline
\end{tabular}

Cadiot (1997), avec aurait un sens primitif de « parallélisme ». Spang-Hanssen, lui, voit en avec un rapport de « symétrie » (1963, p. 239). 


\begin{tabular}{|c|c|c|c|c|}
\hline $\begin{array}{l}\text { Portée } \\
\text { syntaxique }\end{array}$ & $\begin{array}{l}\text { Valeur } \\
\text { sémantique }\end{array}$ & \begin{tabular}{|l} 
Contraintes \\
contextuelles
\end{tabular} & WITH & WITHOUT \\
\hline \multirow[t]{5}{*}{ Adjoint } & Instrumental & $\begin{array}{l}\text { with / without + } \\
\text { GN inanimé } \\
\text { « concret » } \\
\text { - type de verbe : } \\
\text { verbe dynamique } \\
\text { et agentif (incom- } \\
\text { patible avec les } \\
\text { procès statiques). }\end{array}$ & $\begin{array}{l}\text { He sliced the sa- } \\
\text { lami with a knife. } \\
\text { "Il coupa le sala- } \\
\text { mi en tranches } \\
\text { avec un cou- } \\
\text { teau.» }\end{array}$ & $\begin{array}{l}\text { He sliced the sa- } \\
\text { lami without a } \\
\text { knife. } \\
\text { «ll coupa le sala- } \\
\text { mi en tranches } \\
\text { sans couteau.» }\end{array}$ \\
\hline & Coprésence & $\begin{array}{l}\text { with / without + } \\
\text { GN inanimé } \\
\text { «concret » }\end{array}$ & $\begin{array}{l}\text { She left with her } \\
\text { passport and her } \\
\text { savings. } \\
\text { "Elle est partie } \\
\text { avec son passe- } \\
\text { port et ses écono- } \\
\text { mies. " } \\
\text { My son still } \\
\text { sleeps with his } \\
\text { pacifier. } \\
\text { "Mon fils dort } \\
\text { encore avec sa té- } \\
\text { tine. » }\end{array}$ & $\begin{array}{l}\text { She left without } \\
\text { her passport and } \\
\text { all her savings. } \\
\text { "Elle est partie } \\
\text { sans son passe- } \\
\text { port et ses écono- } \\
\text { mies." } \\
\text { My son now } \\
\text { sleeps without his } \\
\text { pacifier. } \\
\text { "Mon fils dort } \\
\text { désormais sans } \\
\text { sa tétine." }\end{array}$ \\
\hline & Comitatif & $\begin{array}{l}\text { with / without + } \\
\text { GN animé } \\
\text { Sujet animé }\end{array}$ & $\begin{array}{l}\text { He went shopping } \\
\text { with his mother. } \\
\text { "Il est allé faire } \\
\text { les courses avec } \\
\text { sa mère.» }\end{array}$ & $\begin{array}{l}\text { He went shopping } \\
\text { without his mo- } \\
\text { ther. } \\
\text { «ll est allé faire } \\
\text { les courses sans } \\
\text { sa mère.» }\end{array}$ \\
\hline & $\begin{array}{l}\text { Manière } \\
\text { (GP incident au } \\
\text { GV) }\end{array}$ & $\begin{array}{l}\text { with / without + } \\
\text { GN continu } \\
\text { compact « abs- } \\
\text { trait » }\end{array}$ & $\begin{array}{l}\text { He answered with } \\
\text { embarrassment. } \\
\text { «Il a répondu } \\
\text { avec embarras. » }\end{array}$ & $\begin{array}{l}\text { He answered wi- } \\
\text { thout embarrass- } \\
\text { ment. } \\
\text { "Il a répondu } \\
\text { sans embarras. » }\end{array}$ \\
\hline & Cause & $\begin{array}{l}\text { with + GN continu } \\
\text { compact « abs- } \\
\text { trait » } \\
\text { - type de verbe : } \\
\text { verbe non agentif } \\
\text { dont le sujet est } \\
\text { patient. }\end{array}$ & $\begin{array}{l}\text { He was trembling } \\
\text { with fear. } \\
\text { «Il tremblait de } \\
\text { peur.» }\end{array}$ & $\begin{array}{l}\text { Non (perte du } \\
\text { sens causal) } \\
\text { He was trembling } \\
\text { without fear. (in- } \\
\text { terprété comme } \\
\text { relevant de la ma- } \\
\text { nière) } \\
\text { « Il tremblait sans } \\
\text { peur. » }\end{array}$ \\
\hline
\end{tabular}




\begin{tabular}{|c|c|c|c|c|}
\hline $\begin{array}{l}\text { Portée } \\
\text { syntaxique }\end{array}$ & $\begin{array}{l}\text { Valeur } \\
\text { sémantique }\end{array}$ & $\begin{array}{l}\text { Contraintes } \\
\text { contextuelles }\end{array}$ & WITH & WITHOUT \\
\hline & $\begin{array}{l}\text { Subordonnée cir- } \\
\text { constancielle }\end{array}$ & $\begin{array}{l}\text { Proposition } \\
\text { gérondive } \\
\text { (avec ou sans su- } \\
\text { jet exprimé) }\end{array}$ & Non & $\begin{array}{l}\text { He left without } \\
\text { saying a word. } \\
\text { "ll est parti sans } \\
\text { dire un mot. » }\end{array}$ \\
\hline Extraprédicatif & $\begin{array}{l}\text { Valeurs sémanti- } \\
\text { ques de concomi- } \\
\text { tance qui peuvent } \\
\text { se réaliser de ma- } \\
\text { nières diverses } \\
\text { (cause, temps, } \\
\text { condition voire } \\
\text { concession). }\end{array}$ & $\begin{array}{l}\text { Prédication } \\
\text { seconde }\end{array}$ & $\begin{array}{l}\text { a) With John away } \\
\text { there's more } \\
\text { room in the } \\
\text { house. } \\
\text { "Avec John qui } \\
\text { n'est pas là, il y a } \\
\text { plus de place } \\
\text { dans la maison. " } \\
\text { b) With her hus- } \\
\text { band by her side, } \\
\text { she felt depres- } \\
\text { sed. } \\
\text { "Avec son mari à } \\
\text { ses côtés, elle se } \\
\text { sentait dépri- } \\
\text { mée. » }\end{array}$ & $\begin{array}{l}\text { Structure } \\
\text { analogue attestée } \\
\text { mais substitution } \\
\text { difficile: } \\
\text { a) *Without John } \\
\text { away there's } \\
\text { more room in the } \\
\text { house. Mais: } \\
\text { b) Without her } \\
\text { husband by her } \\
\text { side, she felt } \\
\text { depressed. }\end{array}$ \\
\hline
\end{tabular}

\subsection{Without ne peut introduire un élément de valence}

Qu'il s'agisse de sans (comme l'ont signalé Choi-Jonin et Mignon [2010]) ou de without, il apparaît que la préposition semble inapte à introduire un élément de valence. Il faudra donc avoir recours à with no ou not... with any... si l'on veut faire porter la négation exclusivement sur cet élément. C'est par exemple le cas avec les verbes transitifs indirects (cope, deal, comply, meet...) : She didn't comply with his demand (et non *She complied without his demand). Ceci n'est pas vraiment étonnant dans la mesure où dans ces constructions, le paradigme est clos : l'emploi de la préposition est figé, difficilement analysable (en synchronie du moins) et son apparition ne semble donc pas sémantiquement motivée. On peut malgré tout déceler un sens instrumental avec certains verbes ditransitifs (provide, mix, load...) : ces verbes semblent d'ailleurs moins réfractaires à la substitution ( ?I mixed the sugar without the eggs). L'impossibilite de trouver without avec des verbes réciproques (fight, quarrel, argue...) pourrait par exemple s'expliquer par le sens adversatif originel de wið, qui faisait bien entendu défaut à wiðutan (voir §3).

On distinguera, lorsque with introduit des GP postnominaux, les cas des adjoints qui autorisent la substitution ( $a$ man with / without a beard) et qui sont 
paraphrasables par not have, des compléments des noms déverbaux (my disappointment with / *without the result) qui l'autorisent plus difficilement.

Pour ce qui est de l'expansion adjectivale, il est à noter qu'il s'agit souvent de participes adjectivaux qui peuvent se construire avec by (satisfied / covered / disappointed...). Nous sommes dans une configuration qui n'est donc pas sans rappeler celle de la phrase passive. Le complément de l'adjectif est donc bien un élément de type valenciel puisqu'il s'agit de mentionner, comme le ferait « un complément d'agent », l'argument source (I was satisfied with the dinner = The dinner satisfied me). Cela n'aurait donc aucun sens de nier son existence.

On pourrait compléter la liste. Cependant, battre en touche en prétextant le figement ou proposer autant d'explications qu'il y a d'interprétations sémantiques à la présence de with dans ces constructions ne nous semblent pas être des solutions satisfaisantes. À ce stade, notre hypothèse sera la suivante : les deux prépositions n'interviendraient pas au même stade dans la genèse de l'énoncé. Si with est apte à participer à la construction de la relation prédicative, c'est-à-dire la sélection et la hiérarchisation des arguments autour desquels l'énoncé s'organise, without, elle, marquerait une opération seconde qui participerait à la finalisation de l'énoncé (validation / non-validation / validabilité ou détermination). Ceci cadre bien avec la valeur négative de l'opérateur vue plus haut (la négation est selon Culioli une modalité de type 1 ; il s'agit donc d'une détermination énonciative qui intervient sur une relation prédicative déjà constituée mais non encore repérée par rapport à une situation) ou avec la présupposition.

\subsection{Without et les causes négatives}

On remarquera en outre qu'en position d'adjoint, l'emploi le plus réfractaire à la substitution est celui du with causal. À dire vrai, without et l'expression de la cause ne semblent pas faire bon ménage. Ceci est vérifiable dans différents emplois que nous allons maintenant examiner.

\section{a) Les compléments de manière et de cause}

Il existe des types d'énoncés très contraints où with prend nettement une interprétation causale :

(58) She shook with emotion. (= She shook because she was moved / because of her emotion.)

« Elle tremblait d'émotion. » (= Elle tremblait parce qu'elle était émue.) 
Cette interprétation n'est possible que lorsque le sujet instancie le rôle thématique d'expérient, tout du moins quand le procès est involontaire. Le nom régime décrit alors un état du référent du sujet qui est la cause de la réalisation du procès. Remplacer with par without ne formerait pas ici un énoncé agrammatical mais verrait la réinterprétation du circonstant de cause en circonstant de manière :

(59) She shook without emotion / shame / fear. (= She showed no emotion while shaking.)

« Elle tremblait sans émotion / honte / crainte. »

Précisons au passage qu'ici, without n'exprime pas vraiment une absence ou un manque. Comme l'a souligné Molinier (1993), les compléments de manière introduits par sans décrivent tout autant des façons d'être que ceux en avec. Without shame est ainsi une manière d'être différente de with shame. Ils peuvent tout autant répondre à la question how ou être remplacés par des adverbes de manière formés à partir d'adjectifs :

(60) 'How did she answer your questions?' 'Without shame and hesitation / Brazenly / Honestly.'

« Comment a-t-elle répondu à vos questions ?

- Sans honte ni hésitation / Effrontément / Honnêtement. »

La relation temporelle impliquée est également différente : là où la cause préexiste nécessairement au procès (l'effet), la manière est de fait concomitante à la réalisation de celui-ci pour la simple raison qu'elle le qualifie :

(61) He answered with / without embarrassment.

"Il a répondu avec / sans embarras.»

His answer was embarrassed / unembarrassed.

«Sa réponse était embarrassée / éhontée.»

Tout semblerait donc indiquer que $\mathrm{X}$ with $\mathrm{Y}$ est apte à exprimer une antériorité notionnelle de $\mathrm{Y}$ par rapport à $\mathrm{X}$, chose que ne peut pas faire $\mathrm{X}$ without $\mathrm{Y}$.

\section{b) L'instrumental}

La plupart des linguistes qui ont étudié l'instrumental postulent que cette relation peut être considérée comme une forme de causation. Talmy a ainsi démontré que 
toute forme de relation instrumentale pouvait être décomposée de la manière suivante :

(caused event) RESULTS FROM (causing event)

where the causing event has the structure: Instrument ACT ON object, where object is bound or related in some way to the object in the caused event (Talmy, 1976).

Il serait donc tout à fait logique que l'emploi de without, parfaitement substituable à with dans ce contexte, exprime lui aussi une forme de causation. Si l'on considère que la relation instrumentale est paraphrasable par le verbe use (Dik, 1997, p. 230), on pourrait reformuler les énoncés (62) de la manière suivante :

(62) a- Ron opened the door with a key.

« Ron a ouvert la porte avec une clef. »

b- He opened the door by using a key.

«Il a ouvert la porte en utilisant une clef. »

c- His using a key caused the door to open.

«Son utilisation d'une clef a entraîné / causé l'ouverture de la porte. »

Or, avec without, cela ne semble pas vraiment être le cas :

(63) a- Ron opened the door without a key.

«Ron a ouvert la porte sans clef.»

b- He opened the door without using a key.

«Il a ouvert la porte sans utiliser de clef.»

$\mathrm{c}-\neq$ ? He opened the door by not using a key.

«? Il a ouvert la porte en n'utilisant pas de clef.»

$\neq$ ? His not using a key caused the door to open.

" ?Sa non-utilisation d'une clef a entraîné l'ouverture de la porte. »

d-He opened the door but he didn't use a key to do it (although I expected him to).

« Il a ouvert la porte mais n'a pas utilisé de clef pour cela (même si je m’y attendais).»

La glose en (63-c), qui aurait bien une valeur causale, n'est pas équivalente à (63a) : dans cet énoncé, la non-réalisation de <he / use a key $>$ déclencherait l'ouverture de la porte, ce qui résulterait en un énoncé douteux ${ }^{7}$. Ce que dit vraiment l'énoncé (63-a), qui est en réalité plus complexe, est restitué en (63-d) :

7 De telles chaînes causales sont pourtant possibles avec by not + Ving : By not using a condom you are at risk for other STD's. « En n'utilisant pas de préservatif, vous risquez d'attraper d'autres MST ». 
- une relation causale était attendue, voire jugée nécessaire (l'utilisation de la clef a pour conséquence que Ron ouvre la porte). On retrouve là la valeur présupposante de without ;

- cette attente ne s'est pas réalisée : Ron a utilisé un autre instrument (voire aucun instrument);

- le fait que cette chaîne causale n'ait pas eu lieu n’a pas empêché la réalisation de <he / open the door $>$.

Without n'exprime donc ici aucune causation (ni absence de causation). Sa fonction semble plus proche d'un énoncé négatif concessif en although par exemple :

(64) Although he didn't use a key, he opened the door.

«Bien qu'il n’ait pas utilisé de clef, il a ouvert la porte.»

Pour rappel, la concession n'est pas totalement étrangère à la cause : il s'agit pour l'énonciateur de rejeter un lien causal là aussi présupposé entre $q$ et $p$. Dans l'énoncé Bien qu'il soit malade, il a fait cours, on considère comme acquis que le fait d'être malade devrait entraîner l'annulation du cours (ne pas faire cours). Without n'intervient donc pas au niveau de la construction de l'événement, mais est la marque d'un rejet $a$ posteriori de la chaîne causale.

\section{c) Les propositions en without + V-ing intraprédicatives}

En ce qui concerne les constructions à verbes non finis en without + V-ING, la substitution de with est impossible. Malgré tout, il demeure souvent possible de remplacer le GP par une proposition participiale en V-ING :

(65) “What do you expect?" he said, without looking at her.

"À quoi vous attendez-vous ?, dit-il, sans la regarder. »

"What do you expect?" he said, (not) looking at her.

«À quoi vous attendez-vous ?”, dit-il, en (ne) la regardant (pas).»

Comme le rappellent Deléchelle et Popineau (2015, p.11), ce type de structurations évite de spécifier explicitement la relation sémantique entre $q$ et $p$. Elles «constituent plutôt un arrière-plan (temporel ou notionnel) au procès principal ». L'interprétation sémantico-logique ne peut être le résultat que d'un 
processus inférentiel qui dépend du contenu lexical des deux propositions ${ }^{8}$ : il pourra s'agir d'une relation causale, temporelle ou d'« une caractérisation qualitative, parfois sur le mode additif (de type paratactique), sorte d'appendice parenthétique, d'afterthought » (Deléchelle 2004, p. 134). La possibilité d'alterner not + V-ING et without + V-ING dans ce type de contexte montre qu'avec la préposition, le type de relation entre les deux propositions serait également nonspécifié. Toutefois, il existe là encore des cas réfractaires à une telle substitution - lorsque la relation entre $q$ et $p$ est clairement causale, par exemple :

(66) Not having had a shower for two days, I was desperate to get to the bathroom. "N'ayant pas pris de douche depuis deux jours, je voulais à tout prix aller dans la salle de bain. "

${ }^{*}$ Without having had a shower for two days, I was desperate to get to the bathroom.

(67) She fears that, having no children, she will have little family assistance as she ages.

«Elle craint que, n'ayant pas d'enfants, elle aura peu d'aide familiale en vieillissant. »

${ }^{*}$ She fears that, without having any children, she will have little family assistance as she ages.

Dans les cas où la transposition demeure malgré tout possible, le sémantisme causal inféré devient totalement exclu avec without et est réinterprétable en une relation d'un autre type (temporelle ou concessive, par exemple) :

8 Deléchelle préfèrera ainsi parler de concomitance, qui se définit comme « une relation sémantique non-spécifiée, évoquant des caractéristiques ou une qualification d'un terme ou d'une prédication, sur le mode associatif » (Deléchelle, 2004, p. 104). Selon lui, cette relation peut intervenir à plusieurs niveaux : événementiel (simultanéité) ; syntaxique : prédications secondes ou détachées portant sur un terme ou sur l'ensemble de $p$, avec un fonctionnement intermédiaire entre l'attributif et le circonstanciel mais n'exprimant pas une circonstance particulière ; discursif : prédications fournissant un repérage complémentaire éclairant l'énonciation principale, soit comme point de départ (en antéposition) soit comme prolongement qualificatif ou explicatif dans une relation souvent métonymique (en postposition). 
(68) John, knowing that his wife was expecting a baby, started to take a course on baby care. (= as he knew his wife was expecting a baby) (exemple de Quirk et al.).

«Sachant que sa femme attendait un bébé, John s'est mis à suivre un cours de soins pour nouveau-nés » (= parce qu'il savait que sa femme attendait un enfant).

John, without knowing that his wife was expecting a baby, started to take a course on baby care (= although he didn't know his wife was expecting a baby).

«Sans savoir que sa femme attendait un bébé, John s'est mis à suivre un cours de soins pour nouveau-nés » (= bien qu'il ignorât que sa femme attendait un enfant).

Qu'en est-il maintenant du rapport temporel entre $p$ et $q$ ? Il est intéressant de constater qu'il est rarement marqué, en français comme en anglais. Ainsi, si le procès introduit par without ou par sans est antérieur à celui de la principale, cette antériorité n'est pas nécessairement marquée par un infinitif parfait en avoir / être + participe passé ou par une forme gérondive en having + V-EN :

(69) Il est allé se coucher sans se laver les dents.

Il est allé se coucher sans s'être lavé les dents.

(70) He went to sleep without brushing his teeth.

He went to sleep without having brushed his teeth. (rare)

Ajoutons que le recours au gérondif parfait est très marginal en anglais : sur 40 109 occurrences de without + V-ING relevées dans le COCA, seulement 405 sont des formes au parfait (soit 1\%). La proportion est la même dans le BNC (nous avons dénombré 103 occurrences de without having + -EN sur un total de 7 177, soit environ $1,5 \%$ ). Il semblerait donc que ce qui importe aux yeux de l'énonciateur, c'est que le procès repère soit faux au moment où celui de la principale a lieu, pas s'il aurait dû ou aurait pu se dérouler avant, pendant ou après ce dernier.

\section{e) Les structures extraprédicatives}

On appellera structure interpropositionnelle, extraprédicative ou encore détachée, un type de prédication seconde (parfois appelé supplementation clauses en 
anglais) ${ }^{9}$. Si de telles structures introduites par with ou avec ont été fort bien décrites (par Ruwet, Cadiot ou Choi-Jonin pour le français, par McCawley en anglais, ou plus récemment par Deléchelle et Popineau dans une perspective contrastive), celles en without, sensiblement plus rares, n'ont fait l'objet que de remarques éparses. Quoi qu'il en soit, les deux types de prédications présentent des propriétés sémantiques, structurelles et discursives analogues. L'exemple suivant, cité par McCawley (1983, p.272), visant à illustrer le phénomène de right node raising, tend à suggérer que les deux structures sont susceptibles de fonctionner en parallèle :

(71) I wouldn't want to live in Sicily with, or for that matter, even without, Mt. Etna erupting. (exemple de McCawley)

« Je ne voudrais pas vivre en Sicile avec - ou même sans, d'ailleurs - l'Etna en éruption. »

(72) Without or even with my husband by my side, I feel depressed.

«Sans, ou même avec, mon mari à mes côtés, je me sens déprimée. »

On dira d'un constituant qu'il est extraprédicatif s'il échappe à la rection d'un terme de la prédication principale, en particulier du verbe. Cela signifie que la relation prédicative principale est construite indépendamment de lui. Par ailleurs, ces structures en with et without ont la particularité d'être des prédications secondes. En surface, on pourra ainsi rencontrer les séquences suivantes :

- with / without GN

- with / without GN + relative réduite en V-ING

- with / without GN GAdj.

- with / without GN + relative infinitive

- with / without GN Adv.

- with / without GN + relative réduite en V-EN

- with / without GN GN

- with / without GN GP

Ainsi, il n'est pas toujours facile de distinguer en surface certains énoncés extraprédicatifs d'autres énoncés intraprédicatifs (notamment quand on aura la structure with / without + GN, ou with / without + GN + GP). Plusieurs tests syntaxiques

9 « We use the term supplementation for a construction containing an anchor and a supplement, an element related semantically to the anchor but not integrated into the syntactic structure as a dependent. Supplements are detached prosodically from the anchor, typically having the character of an interpolation or an appendage (an element added loosely at the beginning or end of a clause) » (Huddleton et Pullum, 2002, p. 66). 
peuvent nous y aider, comme par exemple l'impossibilité de focaliser l'élément introduit par with / without dans une phrase clivée en it :

(73) She spent the rest of her life without her husband by her side (intraprédicatif). «Elle passa le reste de sa vie sans son mari à ses côtés. » Clivée: It was without her husband by her side that she spent the rest of her life.

« C'est sans son mari à ses côtés qu'elle passa le reste de sa vie. »

(74) Without her husband by her side, she felt depressed (extraprédicatif). «Sans son mari à ses côtés, elle se sentait déprimée. » Clivée : ??It was without her husband by her side that she felt depressed. «??C'est sans son mari à ses côtés qu'elle se sentait déprimée. »

La focalisation du constituant introduit par without dans l'énoncé (74) ne nous semble pas totalement impossible mais dans ce cas, il perd sa valeur causale au profit d'une lecture intraprédicative comitative (Her husband was absent when she felt depressed).

Sémantiquement, on retrouve par ailleurs les mêmes effets de sens qu'avec with. Ainsi, comme le relèvent Quirk et al. (1985, p. 1090), cette construction est particulièrement apte à exprimer une condition négative, comme l'illustrent les gloses ci-dessous :

(75) Without me to supplement your income, it wouldn't have happened (exemple de Quirk et al.)

«Sans moi pour compléter ton revenu, cela ne se serait pas produit. » Glose : If I hadn't supplemented your income, it wouldn't have happened. «Si je n'avais pas complété ton revenu, cela ne se serait pas produit.»

(76) Without parents to help them sort out and understand scary news, children are vulnerable to misunderstanding. (COCA)

«Sans des parents pour les aider à faire le tri parmi des informations effrayantes et à les comprendre, les enfants sont exposés à des malentendus. »

Glose : If children don't have parents to help them sort out and understand scary news, they are vulnerable to misunderstanding.

"Si les enfants n'ont pas de parents pour les aider à faire le tri parmi des informations effrayantes et à les comprendre, ils sont exposés à des malentendus. » 
(77) You agree that the world would be better off without him in power. (COCA) " Tu conviendras que le monde se porterait mieux sans lui au pouvoir. » Glose : The world would be better off, if he weren't in power. "Le monde se porterait mieux, s'il n'était pas au pouvoir. »

Toutefois, une condition n'est jamais en définitive qu'une forme de causation (certes hypothétique), puisque l'apodose est la conséquence de la protase. Toutes ces prédications secondes ne sont du reste pas nécessairement hypothétiques, comme le montrent les exemples suivants :

(78) Without her glasses she looked like Master Shifu from Kung Fu Panda. «Sans ses lunettes, elle ressemblait à Maître Shifu dans Kung Fu Panda. » Glose : Because / when ${ }^{10}$ she didn't wear her glasses, she looked like Master Shifu from Kung Fu Panda.

«Quand / Parce qu'elle ne portait pas ses lunettes, elle ressemblait à Maître Shifu dans Kung Fu Panda. »

(79) Without us to remind him, those bad memories disappeared. (COCA) "Sans nous pour les lui rappeler, ces mauvais souvenirs ont disparu. " Glose : Because we didn't remind him, those bad memories disappeared. «Parce que nous ne les lui avons pas rappelés, ces mauvais souvenirs ont disparu. »

(80) Without a taxi soon enough available, Dr Lowe was unable to catch the train. (exemple donné par Quirk et al.)

«Sans taxi disponible suffisamment tôt, le docteur Lowe n’a pas pu avoir son train. »

Glose : Because no taxi was soon enough available, Dr Lowe was unable to catch the train.

« Parce qu'aucun taxi n'était disponible suffisamment tôt, le docteur Lowe n’a pas pu avoir son train.»

10 Les gloses proposées ne suggèrent en aucun cas que les deux structures sont totalement équivalentes ou interchangeables. Elles ne visent qu'à expliciter le lien sémantico-logique sous-jacent entre $q$ et $p$. 
(81) He felt naked without his knife in his pocket.

"Il se sentait nu sans son couteau dans sa poche."

Glose : Because his knife was not in his pocket, he felt naked.

«Parce que son couteau n'était pas dans sa poche, il se sentait nu. »

Plus rarement, le lien entre $q$ et $p$ peut être de nature concessive :

(82) Without money, he is happy.

"Sans argent, il est heureux.»

Glose : Although he has no money, he is happy.

«Bien qu'il n'ait pas d'argent, il est heureux. »

Dans certains cas, il n'est pas possible de déterminer avec précision le rapport logique entre $q$ et $p$ :

(83) Without my husband by my side, I feel depressed.

Glose (a) : If my husband is not by my side, I feel depressed. (lien hypothético-déductif entre $q$ et $p$ )

«Si mon mari n'est pas à mes côtés, je suis déprimée. »

Glose (b) : When my husband is not by my side, I feel depressed. (lien temporel)

«Lorsque mon mari n'est pas à mes côtés, je suis déprimée. »

Glose (c) : Because my husband is not by my side, I feel depressed. (lien causal)

«Parce que mon mari n'est pas à mes côtés, je suis déprimée. »

L'exemple ci-dessus suggère que, comme c'est le cas avec les prédications introduites par with, les rapports sémantico-logiques entre $q$ et $p$ sont extrêmement divers (voire parfois contradictoires), tellement variés qu'il n'est d'ailleurs pas possible, selon de nombreux auteurs, d'assigner à ces constructions une valeur bien définie.

C'est là précisément le rôle de with, qui opère une « association-dissociation » (souvent paraphrasable par have), contrairement à un autre opérateur de concomitance tel que as (qui, lui, opère " une identification plus ou moins étroite ») (Deléchelle et Popineau, 2015). L’interprétation logico-sémantique s’orientera par inférence vers l'une ou l'autre des valeurs vues plus haut (temporelle, causale, hypothétique ou concessive) en fonction de paramètres contextuels comme, par exemple, le temps, l'aspect ou la modalité du verbe principal : ainsi, la forme modalisée en would dans la principale imposera à l'énoncé une lecture hypothéticodéductive. 
On pourrait se demander comment de telles structures extraprédicatives pourraient être aptes à exprimer la cause alors que les structures intraprédicatives utilisant le même marqueur n'ont pas la possibilité d'exprimer une antériorité notionnelle. Je formulerai l'hypothèse que ce n'est pas without (ni with du reste) qui est responsable du lien causal, mais plutôt le statut extraprédicatif et détaché du constituant introduit par la préposition. Comme on l'a vu plus haut, au niveau de la genèse de l'énoncé, $q$ et $p$ sont deux prédications construites indépendamment. Il n'est, d'ailleurs, pas inintéressant de rappeler que normalement, il ne peut y avoir de phénomène de coréférence stricte, qu'il s'agisse d'une structure en with ou en without :

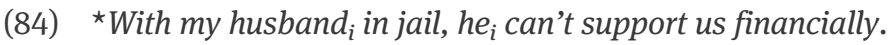

" *Avec mon mari $_{i}$ en prison, $\mathrm{il}_{\mathrm{i}}$ ne peut pas nous aider financièrement. »

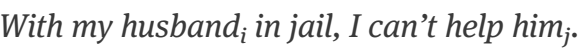

« Avec mon mari $i_{i}$ en prison, je ne peux pas $l_{j}$ 'aider. »

${ }^{\star}$ Without my husband ${ }_{i}$ by my side, $e_{i}$ can't support us financially.

« *Sans mon mari $i_{i}$ à mes côtés, $\mathrm{il}_{\mathrm{i}}$ ne peut pas nous aider financièrement. »

Le GP ne participe donc pas à la construction du sens référentiel de la prédication principale. Il s'agit donc de deux événements bien distincts dont l'association ne va pas de soi, tant sur le plan référentiel, que logique, syntaxique ou discursif. Il n'est donc pas exclu que l'un précède l'autre. C'est ce qui différencie ce type de constituants des GP intraprédicatifs où without indique une circonstance qui ne peut être que simultanée (ou vue de manière simultanée) au procès. With(out) aura pour rôle, dans ces constructions détachées, de mettre en relation $q$ et $p$, et de marquer ainsi de façon explicite, voire iconique, ce lien, sans pour autant avoir de valeur sémantico-logique bien définie.

\subsection{Les structures copulatives : localisation vs. attribution de propriété}

Dans une précédente étude (Gatelais, 2010), j’avais tenté de montrer que, bien que la préposition with ne soit pas considérée traditionnellement comme une préposition spatiale, une valeur locative de proximité ${ }^{11}$ est encore palpable dans certains de ses emplois, en particulier quand elle est employée dans les constructions

11 Sens de proximité suggéré par l'étymologie de avec en français, du latin apud hoc (= près de cela). 
copulatives en be. On pourrait par conséquent s'attendre à ce qu'il soit possible de remplacer dans le même contexte with par without, où cette dernière aurait un sens spatial de «non-proximité » (et donc d'éloignement). Or, cette transformation s'avère normalement impossible :

(85) My keys are with (= next to / close to / near...) your glasses ${ }^{12}$.

«Mes clefs sont avec (= à côté de / près de) tes lunettes. »

${ }^{*}$ My keys are without your glasses.

" ${ }^{\star}$ Mes clefs sont sans tes lunettes. »

Dans le cas où le régime renvoie à un animé, la commutation est en revanche possible :

(86) Sam is with / without his mother.

«Sam est avec / sans sa mère. »

Without semble à première vue nier la proximité spatiale des deux référents (Sam is without his mother =Sam is not near his mother). Pour autant, comme le soulignent Choi-Jonin et Mignon (2010, p. 255), la préposition avec a la capacité de répondre à la question où ? en français, chose que ne peut pas faire sans. Il en va de même en anglais :

(87) Where is Sam? He's with his mother / * without his mother. « Où est Sam ? Il est avec / *sans sa mère. »

De plus, les GP en without, contrairement à ceux introduits par with, ne peuvent apparaître en tête des phrases à inversion sujet-verbe ; or, cette place topicalisée est souvent celle d'éléments locatifs ou directionnels (adverbes ou GP) :

(88) It was then the first night of the second moon, and at the palace gates Lien found the faithful white snake and her other animal friends. But they were not alone. With / * without them were a woman and a man.

«C'était la première nuit de la seconde lune, et aux portes du palais, Lien trouva le loyal serpent blanc et ses autres amis animaux. Ils étaient accompagnés d'un homme et d'une femme » (mot à mot : avec eux / *sans eux étaient un homme et une femme).

12 Pour que cette construction soit possible, il faut que les deux éléments soient amovibles, ce qui explique l'impossibilité de : ${ }^{\star}$ The city hall is with the church. 
(89) With him / * without him was a girl with soft uncoloured hair, springy like heather, and the fresh complexion of someone who spends lots of time outdoors. «Il était accompagné d'une fille aux cheveux soyeux d'une couleur naturelle, souples comme de la bruyère, et qui avait le teint frais d'une personne qui passe beaucoup de temps dehors. »

(90) With / * without the liberation of Rome came a change of government.

«Avec / *sans la libération de Rome est survenu un changement de gouvernement. »

Les deux relations semblent donc de natures différentes et tout semble montrer que without n'est pas la trace d'une relation spatiale ou du moins localisante. Dans d'autres contextes, notamment quand la relation est paraphrasable par not have, l'emploi de without est tout à fait courant :

(91) She was without oxygen / ?with oxygen.

«Elle était sans oxygène / ?avec de l'oxygène. »

(92) She's been without / with her medications for three days.

«Voilà trois jours qu'elle est sans / avec ses médicaments. »

(93) He was without / ?with health insurance.

«Il était sans assurance santé / ?avec une assurance santé. »

(94) He was without teeth, which caused him to look older than he was. I never saw him when he had teeth. (Doyle W. Williams, My Mother's Branch) (?he was with teeth)

«Il était sans dents, ce qui le faisait paraître plus vieux qu'il n'était. Je ne l'ai jamais vu avec des dents.»

Si la substitution est en théorie acceptable dans ces énoncés, les structures en with demeurent relativement gauches (on aurait ainsi tendance à leur préférer le verbe have, marqueur de différenciation et de localisation). Quoi qu'il en soit, dans ces énoncés, l'emploi de without semble moins indiquer un éloignement (une non-proximité) que commenter l'état du référent du sujet sur un mode inférentiel. She was without oxygen implique qu'elle était dans l'incapacité de respirer, to be without her medications indique que son état de santé était fragilisé et susceptible de se dégrader. L'anglais a d'ailleurs parfois la possibilité de lexicaliser cette relation au moyen d'un adjectif dérivé d'un nom en -less : she was toothless. À l'inverse, ?she was with her medications suggèrerait qu'elle avait ses 
médicaments sur elle, ?she was with oxygen qu'elle avait près d'elle une bombonne d'oxygène et ?she was with teeth qu'elle avait des dents en sa possession.

Desclés (1990), dans son analyse célèbre du verbe être, reconnaît quatre valeurs à la copule :

Paris est la capitale de la France.

Identification (caractérisée par la symétrie et la réciprocité)

Paris est une grande ville. Attribution

La France est en Europe. Localisation Les hommes sont mortels. Inclusion entre classes

Les énoncés en be with relèveraient donc de la localisation, tandis que les énoncés en be without relèveraient davantage de l'attribution.

\subsection{Bilan : la valeur de la négation}

$\mathrm{Au}$ terme de cette étude, un faisceau de propriétés différentielles entre with et without a été mis au jour. Ces propriétés infirment l'idée selon laquelle ces deux prépositions sont des complémentaires stricts et confirment donc qu'elles sont les traces d'opérations distinctes. On ne peut donc pas concevoir cette opposition comme relevant d'un côté de la simple «présence » et de l'autre, de la négation de cette présence, de l'absence.

Tableau 3 : Propriétés différentielles de with et without

\begin{tabular}{|l|l|}
\hline WITH & WITHOUT \\
\hline Polarité positive & Polarité négative \\
\hline Association & Dissociation, disjonction \\
\hline $\begin{array}{l}\text { Apte à participer à la construction de la } \\
\text { relation prédicative }\end{array}$ & Participe à la construction de l'énoncé \\
\hline $\begin{array}{l}\text { Susceptible d'exprimer une antériorité notion- } \\
\text { nelle et une relation causale }\end{array}$ & $\begin{array}{l}\text { Inapte à exprimer une antériorité notionnelle et } \\
\text { une cause. Relation temporelle de simultanéité } \\
\text { pertinente ou non }\end{array}$ \\
\hline Relève davantage de la localisation & Relève davantage de l'attribution de propriété \\
\hline $\begin{array}{l}\text { Non-présupposant, pose une relation qui ne } \\
\text { peut être nécessaire }\end{array}$ & $\begin{array}{l}\text { Rejet d'une présupposition, d'une relation pré- } \\
\text { construite dans le discours ou attendue }\end{array}$ \\
\hline
\end{tabular}

Ces conclusions sur la préposition with rejoignent celles de Lise Hamelin, formulées dans le cadre de la Théorie des Opérations Prédicatives et 
Enonciatives d'Antoine Culioli. Selon elle, with est ainsi opérateur de différenciation, qui « opère, au niveau qualitatif, en construisant des propriétés différentielles de l'occurrence $\mathrm{X}$ par le biais de sa mise en relation avec $\mathrm{Y}$, et au niveau quantitatif, en limitant la mise en relation de $\mathrm{X}$ et de $\mathrm{Y}$ à une situation particulière ou à une classe de situations " (Hamelin, 2013; c'est moi qui souligne). Les notions de "différenciation », de "propriétés différentielles » et de «mise en relation à une situation particulière » de ce cadre théorique font écho à ce que nous appelons «association» et «localisation». À l'inverse, without aurait une valeur essentiellement qualitative : ce qui importe n'est pas qu'il existe ou qu'il n'existe pas à un moment donné une occurrence particulière de l'événement qu'elle dénote, mais plutôt le fait que cet événement constitue un cadre, un prérequis notionnel à autre chose. La composante négative peut avoir des portées très diverses, comme on l'a vu :

- (a) il peut s'agir de dire que la relation prédicative introduite par la préposition est fausse (dans le cas des propositions non finies et des structures extraprédicatives), dans ce cas without marque une circonstance, certes négative, mais une circonstance tout de même ;

- (b) la négation peut être de nature plus lexicale, comme c'est le cas avec les compléments de manière : il ne s'agit pas de nier l'existence de cet état mais de renvoyer à un état autre, antonyme (without hesitation = spontaneously, unhesitatingly);

- (c) dans les domaines de l'instrumental et du comitatif, il s'agit du rejet d'un scénario attendu.

À dire vrai, without illustre bien la polyfonctionnalité de la négation que Culioli avait déjà fort bien résumée : "la négation, c'est à la fois le rejet, l'absence, l'altérité, le vide, l'absurde, l'impossible » (Culioli, 1990, p. 84). Dans tous les cas, il nous semble juste que la dimension négative de without relève plus de l'altérité ( $a$ et b) et du rejet (c) que de l'absence.

Ceci rejoint également les conclusions de Choi-Jonin et de Mignon (2010, p. 264) qui voient dans la préposition sans un marqueur de négation discordantielle (notion que l'on doit à Damourette et Pichon). Selon les auteurs, la préposition française serait avant tout un marqueur d'altérité qui « met en opposition une situation privée de l'entité représentée par le terme qu'il introduit, avec une autre situation qui lui sert de caution ». Sans intégrerait ainsi un ne explétif qui peut parfois réapparaître dans les propositions introduites par sans que. Aussi séduisante soit-elle, une telle formulation n'est pas possible en anglais, qui ne connaît pas le ne explétif et encore moins de marqueurs distincts pour le discordantiel et le forclusif. On retiendra malgré tout que l'emploi de without est la trace d'un débat entre deux situations inférées à polarités opposées : celle à polarité négative 
$(\neg p)$ qui est effective, et son complémentaire à polarité positive qui est simplement envisagé, décroché du réel, mais correspondant à l'état attendu des choses.

\section{Retour vers le spatial}

En anglais, la tentation de voir en without un « antonyme » ou un « alter-ego négatif » de with est d'autant plus grande que sa morphologie (with + out) semble nous y inviter. La linguistique diachronique nous enseigne qu'il faut bien souvent se garder de se fier aux apparences. Le cas du couple with / without, apparu au cours de la période moyen-anglaise, est à ce titre un cas d'école, tant ce faux air de famille est le résultat d'un parcours diachronique chaotique. Il ne sera pas question ici de voir en détail comment et pourquoi without s'est imposé (cette question diachronique pourra faire l'objet d'un travail de recherche futur). Revenir sur l'histoire de ces deux formes nous invite plutôt à soulever le problème de l'origine spatiale de ces deux marqueurs, et de ce fait, à nous interroger sur les rapports qu'entretiennent le manque, la relation abessive et l'espace.

\subsection{Destins croisés}

À l'origine, le champ notionnel de l'instrumental / comitatif / manière était pris en charge par la préposition mid, dont les formes apparentées sont encore en usage dans les autres langues germaniques (mit en allemand, med dans les langues scandinaves, met en néerlandais...) et qui sera à terme totalement évincée par la préposition wið dont le sens était adversatif en vieil-anglais (against) ${ }^{13}$. L'abessif était, quant à lui, exprimé par buton, préposition spatiale à l'origine qui signifiait «à l'extérieur de » (be + utan) et qui a elle-même remplacé, à une période préhistorique, la préposition issue du germanique commun ${ }^{\star} e \overline{n u}$ (> allemand ohne). Par la suite, elle développa un sens exclusif / exceptif (emploi encore vivant de nos jours : There was nothing on the table but tea / Il n'y avait rien sur la table à part du thé) puis abessif, pour finalement, à l'époque du moyen-anglais, être réanalysée en conjonction de coordination marquant l'opposition ou la contradiction (> but). Without est attestée dès le vieil-anglais, et était, à l'instar de buton, une préposition spatiale signifiant « outside », dérivée de l'adverbe utan.

13 Pour plus de détails sur le remplacement de mid par wið, voir Gatelais (2010) ou Groussier (2000). 
(95) Ofslogon hyne 7 awurpon wið-utan pone win-geard. (Mathieu 21:39)

" Ils le tuèrent et le jetèrent en dehors de la vigne. »

(96) And pi ilcan geare se cyng Willelm gefeaht togeanes his sunu Rotbearde wiðutan Normandige be anum castele Gerborneð hatte. (Chroniques de Peterborough, année 1079)

« Et la même année, le roi Guillaume combattit son fils Robert en dehors de la Normandie, près d'un château appelé Sherborne. »

Ce sens spatial initial reste attesté en anglais contemporain, uniquement lorsque without est coordonné avec within :

(97) I was awakened by screams and shouts from within and without the house. (COCA)

" Je fus réveillé par des hurlements et des cris qui venaient de l'intérieur et de l'extérieur de la maison. »

With- est donc bien ici un préfixe. Comme le souligne Bourdin (1997, p. 248), son rôle exact est débattu. Pour Mustanoja, il s'agirait d'un intensificateur qui procéderait à un surcodage du sens spatial initial de innan / utan (Mustanoja, 1960, p. 421). Pour Groussier ${ }^{14}$, il s'agirait plutôt d'un préfixe transitivant qui aurait une fonction métagrammaticale et ancrerait innan et utan, deux adverbes (catégorie intransitive), dans la classe des prépositions (catégorie transitive). Wið- aurait donc un rôle analogue au préverbe et préfixe be- en vieil-anglais et dans la plupart des langues germaniques. Quoi qu'il en soit, il semble exclu de voir aujourd'hui dans ce morphème préfixé un quelconque sens adversatif (qui reflèterait la valeur encore contemporaine de la préposition vieil-anglaise wið) et encore moins un sens comitatif ou instrumental, qui serait anachronique.

Si l'on se base sur les datations fournies par l'OED, les premiers sens non spatiaux attestés de without furent l'instrumental (Chroniques de Peterborough, Ms. Laud) et la manière (Lambeth Homilies) au xiI ${ }^{\mathrm{e}}$ siècle. C'est à peu près à la même époque (et même quelques décennies plus tard) que les premiers emplois de with instrumentaux, comitatifs et de manière sont attestés. Malgré tout, il faudra encore quelques siècles pour que with s'impose réellement et supplante mid, qui ne disparaîtra définitivement qu'au $\mathrm{xv}^{\mathrm{e}}$ siècle. Les cas de coordination entre mid et without, très fréquents au début de la période moyen-anglaise, tendent à prouver 
que le couple mid / wibutan était bien installé dans la langue et, incidemment, que l'introduction de wibutan précède celle de wip dans le système :

(98) \& gif he moste pa gyt twa gear libban, he hæfde Yrlande mid his werscipe gewunnon wiputan ælcon wæpnon. (Chroniques de Peterborough, année 1087) "S’il lui avait été donné de vivre deux ans de plus, il aurait conquis l'Irlande par son astuce et sans coup férir. »

(99) Theyh we ne bo at one acorde, We m[a] $]_{3}$ bet mid fayre worde Witute cheste and bute fiste Plaidi mid soze \& mid rizte. (The Owl and the Nightingale, $\mathrm{xII}^{\mathrm{e}} / \mathrm{xIII}^{\mathrm{e}}$ siècle) « Même si nous sommes en désaccord, nous ferions mieux de plaider poliment, avec justesse et exactitude, sans querelle ni dispute. »

Il semble donc indéniable que without ne s'est pas développé à partir de with. L'évolution sémantique de without semble en réalité parallèle à celle de buton, avec lequel il partageait la plupart de ses effets de sens - spatial (« outside »), exclusion (« except »), de condition négative (« unless »), abessif... On peut formuler deux hypothèses pour expliquer ce développement parallèle :

- (a) la première hypothèse est que la synonymie spatiale initiale de wibutan avec buton va déclencher de façon quasi immédiate l'interchangeabilité des deux prépositions dans tous les contextes où buton était employée. Cette hypothèse se heurte à plusieurs contre-arguments de poids. Le premier est chronologique : le sens spatial originel de without est attesté dès le $\mathrm{Ix}^{\mathrm{e}}$ siècle, c'est-à-dire à une époque où le développement polysémique de buton était bien engagé et plusieurs siècles avant sa propre diversification. Par ailleurs, il existe certains emplois de buton qui ne sont pas pris en charge par wibutan, en particulier celui de coordonnant (> but, qui va remplacer la conjonction $a c)^{15}$ qui commence à apparaître à la fin de la période vieil-anglaise. L'inverse est tout autant vrai : wiputan a développé des sens qui faisaient défaut à buton, tel que celui d'addition (« besides, in addition to ») extrêmement bien représenté dans le Brut de Layamon par exemple :

15 Buton va du reste se scinder en deux emplois à l'époque du moyen anglais tardif : l'un accentué et dont la voyelle était longue (bout [e]) et l'autre inaccentué, dont la voyelle était brève ( $>b u t)$ et qui survit encore en anglais contemporain. Le premier, aujourd'hui disparu et remplacé par without, se spécialisera dans l'emploi abessif et spatial, le second dans l'emploi coordonnant et exceptif. La disparition de bout est peut-être imputable à son homonymie avec la forme aphérétique de about ('bout), assez courante au xvi ${ }^{\mathrm{e}}$ siècle. 
(100) fif \& fifti kine-lond; he bi-won to his aзere [азеne] hond wið-uten his riche; pe ær læi to Rome. (Layamon's Brut, début du xiII ${ }^{\mathrm{e}}$ siècle). « Il détint entre ses propres mains cinquante-cinq royaumes sans compter le domaine qui auparavant se trouvait à Rome » (traduction de Françoise Alamichel).

Cette hypothèse semble donc $a$ priori à écarter ;

- (b) La seconde hypothèse est celle du développement naturel à partir du sens spatial. Buton et wiputan auraient eu un développement parallèle (ce qui n'exclut pas une interférence partielle), la notion d'extériorité ayant des affinités avec celle de manque et d'absence. Cette thèse reprend la théorie de la primarité du spatial défendue par Marie-Line Groussier dans sa thèse (1984). Ceci est confirmé par la tendance que de nombreuses langues ont de choisir ce type de préposition spatiale comme marqueur de l'abessif: dans les langues slaves par exemples, без / bez proviennent de la racine proto-indoeuropéenne * $b(h)$ eĝh qui signifierait « hors de ». C'est ce qui se produisit également en suédois $(\text { utan })^{16}$.

Groussier et Bourdin (1997) expliquent l'émergence du sens abessif par la métaphore spatialisante : butan indiquerait «expressément que aucune coïncidence n'est censée exister entre le repéré et le repère » (Groussier, 1984, p. 803). Pour Bourdin, « la préposition [...] tend à participer d'une opération binaire entre le tout et le rien, la présence et l'absence » (Bourdin, 1997). Surtout, dans le système des prépositions spatiales, inside / outside est le seul couple de complémentaires stricts non gradables : -inside $\Leftrightarrow$ outside et $\neg$ outside $\Leftrightarrow$ inside. Par ailleurs, on ne peut pas être plus ou moins à l'intérieur ou à l'extérieur de quelque chose. Ceci n'est pas vrai d'autres couples tels que under et on qui ne sont pas des complémentaires ( $\neg$ under X n'implique pas nécessairement on X) ou near / far from qui sont bien des complémentaires ( $\neg$ near $X \Leftrightarrow$ far from $X$ ) mais qui sont scalaires (on peut être plus ou moins proche / loin de quelque chose). Ce couple spatial est donc bien à même d'opposer le tout au rien, l'être et le non-être et donc d'exprimer une forme de négation. Ce n'est pas du reste un hasard si la TOPE assimile l'opération de négation à l'extérieur du domaine notionnel (Marie-Line

16 Une autre tendance répandue consistera à choisir un marqueur exprimant la séparation. C'est ce qui s'est produit dans les langues romanes (français sans, espagnol sin < latin sine < PIE *senequi exprimait une idée de séparation) ou en néerlandais (zonder, aussi issu de ${ }^{*}$ sene et apparenté à l'anglais sunder / asunder / sundry). 
Groussier précise que métaphoriquement, «l'extérieur du repère, c'est son complémentaire [...] au sens où Culioli l'entend », 1984) ${ }^{17}$.

\subsection{Le sens exceptif : le chaînon manquant ?}

C'est sans doute le sens exceptif (X except / but $\mathrm{Y}$ ) commun à buton et wibutan qui est le « chaînon manquant » entre le rapport spatial d'extériorité et l'abessif. Deux propriétés communes de ces deux emplois nous ont amené à cette conclusion :

- (a) Comme le rappellent Garnier et al. (2009, p. 181), dans ce type d'emplois, l'énonciateur pose d'abord un ensemble, qui doit avoir un caractère « totalisant » (en termes positifs de « tout » ou en termes négatifs de « rien »). Selon les auteurs, « toute évocation d'une situation non absolue, c'est-à-dire toute position intermédiaire entre les pôles du TOUT et du RIEN est réfractaire » à ce type de complémentation. Ceci nous renvoie au caractère absolu lui aussi de l'opposition spatiale intérieur / extérieur vue plus haut ;

- (b) toujours selon Garnier et al., un énoncé du type I had cared for everyone but her serait en réalité un condensé de deux prédications à polarités inverses : l'une positive portant sur la totalité (I had cared for everyone) et l'autre négative portant sur un élément de cette totalité (I had not cared for her). Précisons que de ces deux prédications, seule la seconde est véritablement effective, la première étant seulement envisagée dans un premier temps puis invalidée par le GP introduit par but dans un second temps sur un mode correctif. Ceci rappelle la valeur d'altérité, de débat de validité entre deux prédications, l'une présupposée, l'autre pas, que nous avons pu constater dans l'emploi de la préposition without.

Le passage du sens exceptif au sens abessif correspondrait à un élargissement considérable de la portée du marqueur : là où l'exceptif n'a qu'une portée restreinte sur un constituant nominal qui a un caractère totalisant, l'abessif, lui, portera sur un nom, un prédicat, une proposition entière, un scénario événementiel, etc.

17 Bourdin reconnaît toutefois une inversion des rapports entre repère et repéré avec l'abessif : dans I can manage alright without a car, on pourrait déceler une opération « consistant à repérer l'objet comme étant extérieur au domaine, spatial, dont je suis le centre » (1997, p. 250). Ce type d'inversion semble fréquent en diachronie, comme nous l'avons vu à différentes reprises dans des travaux de recherche antérieurs (on rencontre un tel phénomène avec off et avec with, voir à ce sujet Gatelais, 2011). 


\subsection{Without vs. out of}

Une autre preuve que la notion d'extériorité est intrinsèquement liée à celle de manque est le développement d'une autre structure prépositionnelle concurrente à be without : to be / run out of X.

(101) I'm out of idea / shampoo / milk.

« Je suis à court d'idées / de shampooing / de lait. »

Out of est apparu bien plus tardivement puisque l'OED ne le recense qu'à partir du $\mathrm{xvI}^{\mathrm{e}}$ siècle. On la retrouve ainsi chez Shakespeare :

(102) These English are shrowdly out of Beefe. (Henry V)

«Ces Anglais sont terriblement à court de bœuf. »

Par ailleurs, avec ce sens de manque ou d'absence, la préposition a une distribution bien plus restreinte que celle de without puisqu'elle ne peut apparaître qu'après un verbe d'état (essentiellement be), le verbe run ou en position postnominale (a car out of gas). Le régime de out of est également contraint puisqu'il ne peut s'agir que d'un inanimé, à quelques rares exceptions :

(103) And in a high-profile case with a sequestered jury, we might run out of jurors and then a mistrial will have to be declared. (COCA)

« Et dans une affaire très médiatisée avec un jury mis au secret, nous pourrions être à court de jurés et le procès devra être annulé. »

De plus, elle n'admet qu'une détermination indéfinie (le déterminant $\emptyset$ est en général de règle ou plus rarement l'article $\mathrm{A}(\mathrm{N})$ : How long have you been out of $a$ job? Depuis combien de temps êtes-vous sans emploi ?). En outre, pour que cette construction apparaisse, l'état de manque ne peut être permanent mais doit être momentané :

(104) ??I can't hire her: she is out of experience.

«??Je ne peux pas l'engager : elle est à court d'expérience. »

Sémantiquement, enfin, out of implique que le référent du sujet (siège $d u$ manque) était en possession de l'item manquant mais qu'il ne l'est plus du tout (contrairement à short of qui implique qu'une certaine quantité demeure en sa possession, voir à ce sujet l'étude de V. Hugou dans le présent volume). 
En réalité, cette construction n'est pas sans rappeler l'instrumental, puisqu'elle présuppose un procès visé à valider que l'on pourrait exprimer dans une proposition infinitive de but introduite par (in order) to (105) ou bien une proposition relative infinitive (106)... L'item manquant est alors indispensable pour sa réalisation :

(105) And I see today that they've run out of money to buy grain and so will have to cut back on meat. (COCA)

«Et je m'aperçois aujourd'hui qu'ils manquent d'argent pour acheter des céréales et donc qu'ils seront obligés de réduire leur consommation de viande. »

(106) They're running out of things to talk about. (COCA)

«Ils sont à court de choses à dire. »

Out of n'est donc pas présupposant comme l'est without.

Toutes ces remarques font écho à l'analyse de Lise Hamelin qui oppose out of à from. Selon elle, le lien causal qu'établit out of, opérateur de rupture, semble ne pouvoir être que situationnel : «le marqueur ne peut construire de relation causale qu'entre des termes n'entretenant $\boldsymbol{a}$ priori aucun lien notionnel, et cette relation est nécessairement repérée par rapport à une situation spécifique et à un sujet spécifique ". Hamelin a surtout analysé des énoncés causaux du type She almost fainted out of pain (qui ne sont d'ailleurs pas sans rappeler ceux en with vus plus haut: She fainted with pain). Selon elle, cette relation causale est «non généralisable, non reconstructible à partir des propriétés des termes mis en relation » (Hamelin, 2012) ${ }^{18}$. Ses conclusions nous semblent toutefois tout à fait applicables à la construction copulative be out of X, certes moins orientée vers la causation. On ne retrouve pas cette orientation "situationnelle » et transitoire dans les structures be without $+\mathrm{X}$, qui, elles, sont compatibles avec des procès et des GN génériques :

(107) Ø Red blood cells of all mammals are without nucleus. (Google)

«Les cellules sanguines de tous les mammifères sont dépourvues de noyau. »

L'état dénoté par be without X est donc susceptible d'être permanent. Cette opposition confirme l'orientation qualitative et attributive de la préposition without

18 Elle cite ainsi l'impossibilité : * Divorce often causes death out of love. 
vue plus haut (§ 2.5). Out of aurait dans cet emploi - aussi métaphorique soit-il conservé sa valeur de repérage spatio-temporel : on repère deux entités distinctes, en rupture, dans une situation spécifique sur un mode qui demeure additif. Cette orientation pourrait être confirmée par l'emploi du verbe run, verbe qui dénote à l'origine un mouvement spatial.

Without, à l'inverse, procède par soustraction et correction : on ôte un item à un ensemble préalablement construit sur un mode qualitatif. La valeur spatiale de without est donc réduite en anglais contemporain à peu de choses : elle a fait l'objet d'une réanalyse et d'une extension métaphorique qui ont eu pour conséquence sa grammaticalisation partielle (§3.2). À l'inverse, les affinités plus perceptibles de out of avec l'espace ne devraient pas nous étonner si l'on considère que ses sens spatiaux sont encore bien vivants et même prototypiques, tandis que ceux de without ne sont finalement attestés que dans des contextes figés et fossilisés.

\section{Conclusion}

Nous espérons que cette étude aura comblé un manque dans la description, tant synchronique que diachronique, de la préposition without. Notre ambition n'aura pas été ici de dégager une valeur invariante ou une forme schématique « déformable en contexte » pour cette préposition, mais plus modestement de dégager un faisceau de propriétés différentielles ou partagées (sémantiques, énonciatives, syntaxiques, historiques...) observables en discours avec d'autres marqueurs à première vue opposés (with) ou proches (out of). Dans une recherche future, l'analyse pourrait être affinée par la confrontation avec don't have, les adjectifs dérivés en -less ou composés en -free et surtout with no, qui semble alterner assez librement avec without. Faute de place, il n'a pas été possible de mener ces études.

Nous avons donc tenté de démontrer que without ne pouvait pas être appréhendée simplement comme une "préposition négative », un marqueur de négation, le complémentaire de with ou le constat d'une absence. Comme l'ont suggéré les travaux de Lagacé et de Riegel pour le français, la notion de présupposition, bien que galvaudée en linguistique, et malgré ses avatars très divers dans la littérature, semble un outil particulièrement éclairant, indispensable même, à la compréhension du phénomène et aura donc été le fil conducteur de cette étude. Bien entendu, ce processus ainsi que tous les mécanismes inférentiels qu'il implique dans la construction du sens pourraient faire l'objet d'une étude plus détaillée et plus systématique. 
C'est surtout à cause de cette dimension polyphonique (comme l'a formulé Ducrot, 1984) que without s'inscrit dans l'opération du manque : à l'instar du verbe manquer en français décrit par Alain Delplanque dans le présent volume, la préposition confronte « un état de choses constaté (la situation effective $\mathrm{Sit}_{0}$ ) et un état idéal (une situation fictive Sit' ${ }^{\text {) }}$ ), tel que ce qui aurait pu ou dû être présent ne l'est pas ». Ce qui différencie without de la plupart des verbes et des lexèmes étudiés dans ce volume est la valuation et l'orientation appréciative de l'état résultant (voir à ce sujet les études d'Alain Delplanque, de Sylvester N. Osu et de Patrick Gettliffe qui ont bien rendu compte de ce phénomène) : peut-être est-ce dû au fait que la modalité appréciative (de rang 3, selon Culioli) est moins un phénomène grammatical que lexical, et que la préposition n'est pas une catégorie pleinement signifiante. Notre étude diachronique de without (qu'il sera possible de détailler par la suite) a enfin mis au jour les rapports entre espace, absence et manque, posant ainsi le problème de la métaphore spatialisante dans la langue. Là encore, la problématique prépositionnelle s’y prête particulièrement, ce qui ne semble pas être le cas des verbes étudiés dans ce volume.

\section{Bibliographie}

\section{Sources secondaires}

Adamczewski Henri et Delmas Claude, 1982, Grammaire linguistique de l'anglais, Paris, Armand Colin.

Boulonnais Dominique, 2004, «To et les infinitives : l'hypothèse de la transcendance prépositionnelle », in Delmas C. (dir.), CIEREC Travaux, 116 : La contradiction en anglais, p. 55-90.

Bourdin Pierre, 1997, «À propos des prépositions anglaises within et without : note sur une impossible antonymie », in Danon-Boileau L. et Morel M.-A. (dir.), FDL 9 : La préposition : une catégorie accessoire?, Gap, Ophrys.

Cadiot Pierre, 1997, Les prépositions abstraites en français, Paris, Armand Colin.

Cervoni Jean, 1991, Les prépositions : étude sémantique et pragmatique, Paris/Louvain-la-Neuve, Duculot (Champs linguistiques).

Choi-Jonin Injoo et Mignon Françoise, 2010, « Sans, préposition négative d'avec ? ", Journal of French Studies 20, 3, p. 253-270.

Corblin Francis et Tovena Lucia, 2003, « L'expression de la négation dans les langues romanes », in Godard D., Les langues romanes : problèmes de la phrase simple, Paris, CNRS éditions, p. 279-242.

Culioli Antoine, 1990, Pour une linguistique de l'énonciation, t. I : Opérations et représentations, Paris/Gap, Ophrys.

Crystal David, 2008, A Dictionary of Linguistics and Phonetics, Oxford, Blackwell publishing.

Delechelle Gérard, 2004, «Causalité et phrase complexe : prédications et circonstances concomitantes », Cercles 9, p. 121-142. 
Delechelle Gérard et Popineau Joëlle, 2015, « Les prédications en with : position et sémantisme », Corela [en ligne] 13-1.

Desclés Jean-Pierre, 1990, Langages applicatifs, langues naturelles et cognition, Paris, Hermès. Dik Simon C., 1997, The Theory of Functional Grammar (Part II: Complex and Derived Constructions), Berlin, Mouton de Gruyter (Functional Grammar Series 21).

Dubois Jean et al., 1994, Dictionnaire de linguistique et des sciences du langage, Paris, Larousse. Ducrot Oswald, 1984, Le dire et le dit, Paris, Les Éditions de Minuit.

Feigenbaum Susanne, 1997, « Le mot composé [sans + SN] : ses rapports syntaxiques, sémantiques et morphologiques ", Travaux de Linguistique 34, p. 21-47.

Garnier Georges, Guimier Claude et Dilys Rosalind, 2002, L'épreuve de Linguistique à l'Agrégation d'anglais : grammaire, phonologie, Paris, Nathan/VUEF.

Gatelais Sylvain, 2008, La couleur des prépositions en anglais : l'exemple de with (représentation, sémantaxe, référence), thèse nouveau régime, Université de Paris 3 Sorbonne Nouvelle, sous la direction de Geneviève Girard-Gillet.

Gatelais Sylvain, 2010, « With est-elle une préposition spatiale ? », in Delmas C. (éd.), Espace temps en anglais, Faits de Langues, Paris, Ophrys.

Groussier Marie-Line, 1984, Le système des prépositions dans la prose en vieil-anglais, thèse d'État, tirée en offset.

Groussier Marie-Line, 2001, « Pourquoi la préposition vieil-anglaise MID a-t-elle disparu au profit de WITH ? Arguments en faveur d'une origine cognitive de cette disparition », Graat 24, p. 21-37.

Hamelin Lise, 2012, " Out of et from, une synonymie attendue ? ", Linx [en ligne], p. 66-67.

Hamelin Lise, 2013, «Dealing with with », Syntaxe et sémantique 1, 14, p. 67-83.

Huddleston Rodney et Pullum Geoffrey K., 2002, The Cambridge Grammar of the English, Cambridge, Cambridge University Press.

Jamet Denis, 2008, « Existe-il un invariant sémantique pour le marqueur -ING ? ", Journée Spéciale concours CAPES-Agrégation d'anglais 2008-2009, Université Jean Moulin-Lyon 3, décembre 2008, Lyon, France, [pp.www.cercles.com].

Klima Edward S., 1964, " Negation in English », in Fodor J. A. et Katz J. J., 1964, The Structure of Language. Readings in the Philosophy of Language, Upper Saddle River, Prentice-Hall, p. 246-321.

Lagacé Michel-Francis, 1987, «L'analyse sémantique de sans (que) : retour de la présupposition ?", Revue québécoise de linguistique théorique et appliquée 6, p. 169-182.

Lakoff George et Johnson Mark, 1980, Metaphors We Live By, Chicago, University of Chicago Press.

Lapaire Jean-Rémi et Rotgé Wilfrid, 1992, Réussir le commentaire grammatical des textes anglais (CAPES / Agrégation), Paris, Ellipses.

McCawley James D., 1983, « What's with with », Language 59, p. 271-287.

Molinier Christian, 1993, « Les expressions sans N du français », Cahiers de Grammaire 18, p. 31-72.

Mustanoja Tauno, 1960, A Middle English syntax 1. Parts of speech, Helsinki, Mémoires de la Société néophilologique de Helsinki 23.

Priestley Joseph, 1761, The Rudiments of English Grammar; adapted to the use of schools. With observations on style, Londres, Printed for R. Griffits.

Quirk Randolph et al., 1985, A Comprehensive Grammar of the English language, Londres, Longman.

Riegel Martin et al., 1994, Grammaire méthodique du français, Paris, PUF. 
Riegel Martin, 1977, « La Représentation sémantique de sans que », Travaux de linguistique et de littérature XV, 1, p. 337-359.

Ruwet Nicolas, 1978, « Une construction absolue en français », Linguisticae Investigationes 11, p. $165-210$.

Schlesinger Izchak M., 1979, "Cognitive structures and semantic deep structures: the case of the instrumental », Journal of Linguistics 15, p. 307-324.

Spang-Hanssen Ebbe, 1963, Les prépositions incolores du français moderne, Copenhague, G. E. C. Gads Forlag.

Talmy Leonard, 1976, "Semantic Causative Types », in Shibatani M. (éd.), Syntax and Semantics 6: The Grammar of Causative Constructions, New York, Academic Press, p. 43-116.

Vallins George Henry, 1956, The Pattern of English, Londres, Andre Deutsch Ltd.

Visser Fredericus Theodorus, 1966, An Historical Syntax of the English Language, partie 2 : Syntactical Units with One Verb (Continued), Leyde, Brill.

\section{Sources primaires, dictionnaires et corpus}

Oxford English Dictionary, Oxford, Oxford University Press, 1989.

Merriam-Webster's Advanced Learner's English Dictionary, Springfield (MA), Merriam-Webster, 2008.

Dictionnaire de français Larousse, [https://www.larousse.fr/dictionnaires/francais].

Corpus of Contemporary American English, [ http://corpus.byu.edu/coca].

The Middle English Compendium of the University of Michigan, [http://quod.lib.umich.edu/m/ $\mathrm{mec} /]$.

Saint Exupéry Antoine de, 1943, The Little Prince (traduction de Richard Howard), San Diego (CA), Harcourt. 
